PHYSICAL REVIEW D 86, 074021 (2012)

\title{
Bulk viscosity and the phase transition of the linear sigma model
}

\author{
Antonio Dobado* \\ Departamento de Física Teórica I, Universidad Complutense de Madrid, 28040 Madrid, Spain
}

\author{
Juan M. Torres-Rincon ${ }^{\dagger}$ \\ School of Physics and Astronomy, University of Minnesota, Minneapolis, Minnesota 55455, USA
}

(Received 12 June 2012; published 15 October 2012)

\begin{abstract}
In this work, we deal with the critical behavior of the bulk viscosity in the linear sigma model $(\mathrm{L} \sigma \mathrm{M})$ as an example of a system which can be treated by using different techniques. Starting from the BoltzmannUehling-Uhlenbeck equation, we compute the bulk viscosity over entropy density of the L $\sigma \mathrm{M}$ in the large- $N$ limit. We search for a possible maximum of $\zeta / s$ at the critical temperature of the chiral phase transition. The information about this critical temperature, as well as the effective masses, is obtained from the effective potential. We find that the expected maximum (as a measure of the conformality loss) is absent in the large- $N$ limit in agreement with other models in the same limit. However, this maximum appears when, instead of the large- $N$ limit, the Hartree approximation within the Cornwall-JackiwTomboulis formalism is used. Nevertheless, this last approach to the $\mathrm{L} \sigma \mathrm{M}$ does not give rise to the Goldstone theorem and also predicts a first-order phase transition instead of the expected second-order one. Therefore, both the large- $N$ limit and the Hartree approximations, should be considered relevant and informative for the study of the critical behavior of the bulk viscosity in the L $\sigma \mathrm{M}$.
\end{abstract}

DOI: 10.1103/PhysRevD.86.074021

PACS numbers: $51.20 .+\mathrm{d}, 25.75 .-\mathrm{q}, 25.75 . \mathrm{Nq}$

\section{INTRODUCTION}

Transport properties are essential to understand the equilibration of the hadronic matter created at heavy-ion colliders. In this context, the most studied transport coefficient has been the shear viscosity $\eta$. When two relativistic nuclei collide, the initial spatial anisotropy of the interacting region is converted into momentum anisotropy due to the hydrodynamical evolution. The momentumanisotropy equilibration is mainly controlled by the shear viscosity, and it influences the value of the flow coefficients $v_{n}$. Comparing the measured value of the elliptic flow $v_{2}[1,2]$ with the numerical hydrodynamic simulations, a value of $\eta / s \simeq 0.1$ has been estimated [3-5] (where $s$ is the entropy density). This small value, very close to the Kovtun, Son, and Starinets (KSS) minimum conjectured in Ref. [6], has characterized the matter created in relativistic heavy-ion collisions as a nearly perfect fluid. It has also been shown that the KSS coefficient $\eta / s$ has a minimum at the liquid-gas phase transition of common fluids $[7,8]$. A minimum near the phase transition of the linear sigma model $(\mathrm{L} \sigma \mathrm{M})$ in the large- $N$ limit has also been found [9], and this minimum is also expected in the deconfinement phase transition of QCD $[8,10]$.

Another transport coefficient which relates momentum flux with a velocity gradient is the bulk viscosity $\zeta$. It is sensitive to uniform expansions of the system in such a way that it is closely related to the scale invariance of the

\footnotetext{
*dobado@fis.ucm.es

†jtorres@physics.umn.edu, j.torres@fis.ucm.es

*On leave of absence from Departamento de Física Teórica I, Universidad Complutense de Madrid, 28040 Madrid, Spain.
}

fluid [11,12]. For conformal systems, the bulk viscosity identically vanishes. This coefficient has been assumed to be much smaller than the shear viscosity as is the case for common fluids. Moreover, in perturbative QCD, the ratio between the bulk viscosity and the shear viscosity has been estimated around $10^{-3}-10^{-8}$ [13]. In the low temperature phase, the hadronic medium, this coefficient has been calculated using the Green-Kubo formalism and kinetic theory in Refs. [14,15], respectively. In this phase, the ratio $\zeta / \eta$ has been found to be around $10^{-3}-3 \times 10^{-3}$ [16].

However, near the critical point, $\zeta$ can be larger than the shear viscosity. For those systems belonging to the dynamical universality class of model $\mathrm{H}$ (like the liquid-gas transition), the bulk viscosity diverges [17] with the correlation length as $\zeta \simeq \xi^{2.8}$ (or as a function of the reduced temperature $\left[t=T / T_{c}-1\right]$ as $\zeta \simeq t^{-1.8}$ ). In lattice QCD calculations of the bulk viscosity over entropy density above the critical temperature [18,19], this coefficient seems to diverge at $T_{c}$. Other authors have pointed out that the bulk viscosity presents a maximum in the critical temperature for some different models like the $O(N)$ model, even at mean-field level [20-23]. However, this maximum at $T_{c}$ is not seen in other systems as in the Gross-Neveu model in the limit of a large number of fermion fields [24], where the bulk viscosity is a monotonically decreasing function when temperature increases. In the context of the $O(N)$ model, it has been recently shown [25] that it belongs to model $C$ if $N=1$ and to model $G$ if $N>1$ (particularly, the large- $N$ limit) with a divergence with the correlation length if $N=1$ going as $\zeta \sim \xi^{2}$ and being finite for $N>1$ as $\zeta \sim \xi^{0}$.

In the hydrodynamic calculations of relativistic heavyion collisions, the bulk viscosity over entropy density can 
also be extracted. For example, in Ref. [5], it is found that a value $\zeta / s=0.04$ is compatible with the integrated elliptic flow measured by RHIC and ALICE collaborations. In Ref. [26], a similar value is found $(\zeta / s \lesssim 0.05)$ near the freeze-out temperature.

In principle, our goal is to study the behavior of the bulk viscosity over entropy density in the $\mathrm{L} \sigma \mathrm{M}$ in the large- $N$ limit following the same lines of our previous article [9]. We will compare the phase diagram of the $\mathrm{L} \sigma \mathrm{M}$ in the large- $N$ limit with the coefficient $\zeta / s$ in order to ascertain if a maximum of $\zeta / s$ is found near $T_{c}$. In Sec. II, we present the $\mathrm{L} \sigma \mathrm{M}$ and its effective potential at finite temperature in the large- $N$ limit. In Sec. III, we review the calculation of the shear viscosity over entropy density in this model. In Sec. IV, we will perform the detailed computation of the bulk viscosity over entropy density and discuss the results in terms of the conformality loss of the system. In Sec. V, we repeat the calculation for $\zeta / s$ in the context of the Cornwall-Jackiw-Tomboulis (CJT) formalism in the Hartree approximation. Finally, in Sec. VI, we present our main conclusions.

\section{LoM LAGRANGIAN IN THE LARGE- $N$ LIMIT}

In this section, we will review the dynamics of the $\mathrm{L} \sigma \mathrm{M}$ at finite temperature, deriving the effective potential in the large- $N$ limit. As we have already detailed this procedure in Ref. [9], we will only sketch the key steps of the calculation.

The bare Euclidean Lagrangian of the $\mathrm{L} \sigma \mathrm{M}$ reads [27]

$$
\mathcal{L}=\frac{1}{2} \partial_{\mu} \Phi^{T} \partial^{\mu} \Phi-\bar{\mu}^{2} \Phi^{T} \Phi+\frac{\lambda}{N}\left(\Phi^{T} \Phi\right)^{2}-\epsilon \Phi_{N+1},
$$

where the multiplet $\Phi=\left(\pi_{i}, \sigma\right)(i=1, N)$ contains $N+1$ scalar fields. $\lambda$ is positive in order to have a potential bounded from below, and we consider $\bar{\mu}^{2}$ to be positive in order to provide a spontaneous symmetry breaking (SSB). When $\epsilon=0$, the SSB pattern is $S O(N+1) \rightarrow$ $S O(N)$. In the following, we will refer for historical reasons to the $\pi$ fields as pions (even if it is well established that the $\mathrm{L} \sigma \mathrm{M}$ is not an accurate model for chiral dynamics) and to the $\sigma$ related degree of freedom as the Higgs.

The factor $\epsilon=m_{\pi}^{2} f_{\pi}$ is responsible for the physical pion mass, and, as it is well known, it gives rise to an explicit breaking of the $S O(N+1)$ symmetry. In the limit $T=0$, the potential has a nonzero vacuum expectation value (VEV), and one expects SSB. Choosing the VEV, which will be denoted by $f_{\pi}$, pointing in the $N+1$ direction, we get the equation

$$
-2 \bar{\mu}^{2} f_{\pi}+\frac{4 \lambda}{N} f_{\pi}^{3}-\epsilon=0 .
$$

For small $\epsilon$, the solution to this equation is

$$
f_{\pi}=\sqrt{\frac{N \bar{\mu}^{2}}{2 \lambda}}+\frac{\epsilon}{4 \bar{\mu}^{2}}=f_{\pi}(\epsilon=0)+\frac{\epsilon}{4 \bar{\mu}^{2}} .
$$

The VEV can be written in terms of the $N$-independent $F$ parameter defined as

$$
\left\langle\Phi^{T} \Phi\right\rangle=\left\langle\sigma^{2}(T=0)\right\rangle=f_{\pi}^{2}=N F^{2} .
$$

In our notation, we will call $f_{\pi}(\epsilon=0)$ and $f_{\pi}$ the VEV at $T=0$ for the cases without and with an explicit symmetry breaking term, respectively. In the next sections, the VEV at arbitrary temperature will be denoted by $v(T)$, in such a way that $f_{\pi}=v(T=0)$.

At $T=0$, the low-energy dynamics is controlled by the broken phase. In this case, $\left\langle\pi^{a}\right\rangle=0$. Then, the relevant degrees of freedom are the pions which correspond to the (pseudo) Goldstone bosons when $(\epsilon \neq 0) \epsilon=0$. Fluctuations along the $\sigma$ direction will be denoted by $\tilde{\sigma}$, and they correspond to the Higgs, the massive mode which is relevant at higher energies (or temperatures):

$$
\sigma=f_{\pi}+\tilde{\sigma} \rightarrow\langle\sigma\rangle=f_{\pi} .
$$

The Lagrangian (1) written in terms of $\pi^{a}, f_{\pi}$ and $\tilde{\sigma}$ reads

$$
\begin{aligned}
\mathcal{L}= & \frac{1}{2} \partial_{\mu} \pi^{a} \partial^{\mu} \pi^{a}+\frac{1}{2} \partial_{\mu} \tilde{\sigma} \partial^{\mu} \tilde{\sigma}-\bar{\mu}^{2} \pi^{a} \pi^{a}-\bar{\mu}^{2} \tilde{\sigma}^{2} \\
& +\frac{\lambda}{N}\left[2 f_{\pi}^{2}\left(\pi^{a} \pi^{a}+3 \tilde{\sigma}^{2}\right)+\left(\pi^{a} \pi^{a}\right)^{2}+2 \pi^{a} \pi^{a} \tilde{\sigma}^{2}\right. \\
& \left.+\tilde{\sigma}^{4}+4 f_{\pi} \tilde{\sigma}^{3}+4 \pi^{a} \pi^{a} f_{\pi} \tilde{\sigma}\right] \\
& +\left(-\epsilon+\frac{4 \lambda}{N} f_{\pi}^{3}-2 \bar{\mu}^{2} f_{\pi}\right) \tilde{\sigma} \\
& -\bar{\mu}^{2} f_{\pi}^{2}+\frac{\lambda}{N} f_{\pi}^{4}-\epsilon f_{\pi} .
\end{aligned}
$$

Note that the tadpole term vanishes because of Eq. (2). The tree-level Higgs mass can be read from the Lagrangian in Eq. (6):

$$
M_{\tilde{\sigma}}^{2}=-2 \bar{\mu}^{2}+\frac{12 \lambda}{N} f_{\pi}^{2},
$$

and it can also be written as

$$
M_{\tilde{\sigma}}^{2}=4 \bar{\mu}^{2}+3 \frac{\epsilon}{f_{\pi}}=\frac{8 \lambda f_{\pi}^{2}}{N}+\frac{\epsilon}{f_{\pi}} \simeq \frac{8 \lambda f_{\pi}^{2}(\epsilon=0)}{N}+3 \frac{\epsilon}{f_{\pi}},
$$

using Eq. (3) for the last identity.

The pion mass reads

$$
m_{\pi}^{2}=-2 \bar{\mu}^{2}+\frac{4 \lambda}{N} f_{\pi}^{2}
$$

which, as expected, depends only on the explicit symmetry breaking term

$$
m_{\pi}^{2}=\frac{\epsilon}{f_{\pi}}
$$


and vanishes when $\epsilon=0$ (obviously all pions are degenerate). The pion and Higgs masses are related through

$$
M_{\tilde{\sigma}}^{2}=m_{\pi}^{2}+\frac{8 \lambda f_{\pi}^{2}}{N}
$$

For small enough explicit symmetry breaking, we have

$$
\begin{gathered}
\bar{\mu}^{2}=\frac{M_{\tilde{\sigma}}^{2}-3 m_{\pi}^{2}}{4}, \\
\lambda=\frac{N}{8 f_{\pi}^{2}}\left(M_{\tilde{\sigma}}^{2}-m_{\pi}^{2}\right) .
\end{gathered}
$$

Equation (13) can be expressed in terms of the VEV at $\epsilon=0$ :

$$
\lambda=\frac{N}{8 f_{\pi}^{2}(\epsilon=0)} \frac{M_{\tilde{\sigma}}^{2}-m_{\pi}^{2}}{\alpha^{2}},
$$

where $\alpha$ is a multiplicative constant relating $f_{\pi}$ and $f_{\pi}(\epsilon=0)$,

$$
f_{\pi}=\alpha f_{\pi}(\epsilon=0),
$$

which can be written as

$$
\alpha=\frac{M_{\tilde{\sigma}}^{2}-3 m_{\pi}^{2}}{M_{\tilde{\sigma}}^{2}-4 m_{\pi}^{2}},
$$

where we have used Eq. (3) and consider small $\epsilon$.

In order to obtain the effective potential, we will integrate out the fluctuations from the partition function $Z$. To do that, it is convenient to introduce an auxiliary scalar field. This field allows for a systematic counting of $N$ factors and provides some simplifications in the large- $N$ limit. Then, the integration of the pions and the Higgs is performed by standard Gaussian integration.

The integration of the fluctuations is done regardless of their wavelengths. All the frequency modes of the scalar fields are treated at the same footing, and this "unorganized" integration produces two undesirable features in the effective potential. First, an imaginary part of the effective potential appears. This imaginary part has been given the interpretation of a decay rate per unit volume of the unstable vacuum state by Weinberg and Wu in Ref. [28].

The second characteristic is the nonconvexity of the quantum effective potential. However, the effective potential, defined through a Legendre transformation of the generating functional of the connected diagrams [29], should always be convex. This nonconvexity problem and the imaginary part appear as long as a perturbative method is used to calculate the effective potential [30].

A possible solution for these problems can be given by using a nonperturbative method to generate the effective potential. For example, the functional renormalization group generates an effective potential in such a way that an organized integration of the fluctuations is performed. Following the ideas of the renormalization group, only the low-wavelength components of the quantum fluctuations are integrated out at each step. Thus, the UV components are infinitesimally integrated step by step, and the final effective potential (defined in the infrared scale) does not acquire an imaginary part, and it remains convex at every scale (at the IR point, the Maxwell construction is dynamically generated by the renormalization flow) [31].

In spite of the previous discussion, we understand that it is not necessary to perform a more sophisticated method to obtain the effective potential. The only relevant information for us is the location of the effective potential minimum, which eventually gives the position of the critical temperature. This minimum appears always outside of the nonconvex region. On the other hand, the possible presence of an imaginary part (whose domain in fact coincides with the domain of the nonconvex part of the effective potential) is not relevant for us in this work.

\section{A. Auxiliary field method and effective potential at $T \neq 0$}

To compute the effective potential in the large- $N$ limit, we start by considering the partition function:

$$
\mathcal{Z}=\int \mathcal{D} \pi^{a} \mathcal{D} \sigma \exp \left(-\int d^{4} x \mathcal{L}\right),
$$

with the Lagrangian of Eq. (1). Then, we introduce an auxiliary field $\chi$ in order to deal with the quartic coupling by using the Gaussian integral:

$\exp \left(-\int d^{4} x \frac{\lambda}{N} \Phi^{4}\right) \propto \int \mathcal{D} \chi \exp \left[\int d^{4} x\left(\frac{N}{8 \lambda} \chi^{2}-\frac{\sqrt{2}}{2} \chi \Phi^{2}\right)\right]$

To prove the equivalence between the two Lagrangians, note that this auxiliary field has introduced a mass term and a coupling with $\Phi^{2}$ in the Lagrangian. However, it has no kinetic term which means that $\chi$ has not a true dynamics. The Euler-Lagrange equation for $\chi$ simply gives $\chi=$ $2 \sqrt{2} \lambda \Phi^{2} / N$. Introducing this solution into the right-hand side of Eq. (18), one obtains the Lagrangian

$$
\mathcal{L}=-\frac{N}{8 \lambda} \chi^{2}+\frac{\sqrt{2}}{2} \chi \Phi^{2}=-\frac{\lambda}{N} \Phi^{4}+2 \frac{\lambda}{N} \Phi^{4}=\frac{\lambda}{N} \Phi^{4},
$$

which is the original interaction Lagrangian.

The partition function can then be written as

$$
\begin{aligned}
Z= & \int \mathcal{D} \pi^{a} \mathcal{D} \sigma \mathcal{D} \chi \exp \left[-\int d^{4} x\left(\frac{1}{2} \partial_{\mu} \pi^{a} \partial^{\mu} \pi^{a}\right.\right. \\
& +\frac{1}{2} \partial_{\mu} \sigma \partial^{\mu} \sigma-\bar{\mu}^{2} \pi^{a} \pi^{a}-\bar{\mu}^{2} \sigma^{2}-\frac{N}{8 \lambda} \chi^{2} \\
& \left.\left.+\frac{\sqrt{2}}{2} \chi \pi^{a} \pi^{a}+\frac{\sqrt{2}}{2} \chi \sigma^{2}-\epsilon \sigma\right)\right] .
\end{aligned}
$$


Thus the action in terms of the $\pi^{a}, \sigma$, and $\chi$ fields reads

$$
\begin{aligned}
S= & \int d^{4} x\left[\frac{1}{2} \pi^{a}\left(-\square_{E}-2 \bar{\mu}^{2}+\sqrt{2} \chi\right) \pi^{a}\right. \\
& \left.+\frac{1}{2} \sigma\left(-\square_{E}-2 \bar{\mu}^{2}+\sqrt{2} \chi\right) \sigma-\frac{N}{8 \lambda} \chi^{2}-\epsilon \sigma\right] .
\end{aligned}
$$

Before identifying properly the pion propagator, one must get rid of the unphysical $\sigma$ tadpole. We have already seen that this term vanishes at $T=0$. Now, we perform a shift of the $\sigma$ field $\sigma=v+\tilde{\sigma}$ which also produces a change in the auxiliary field $\chi=\tilde{\chi}+2 \sqrt{2} \frac{\lambda}{N} v^{2}+4 \sqrt{2} \frac{\lambda}{N} v \tilde{\sigma}$ allowing us to cancel the tadpole term for $\tilde{\sigma}$ and the unphysical mass mixing term between $\tilde{\sigma}$ and $\tilde{\chi}$. After some manipulations, we obtain the action

$$
\begin{aligned}
S= & \int d^{4} x\left[\frac{1}{2} \pi^{a}\left(-\square_{E}+G_{\pi}^{-1}[0, \chi]\right) \pi^{a}\right. \\
& +\frac{1}{2} \tilde{\sigma}\left(-\square_{E}+G_{\pi}^{-1}[0, \chi]+8 \frac{\lambda}{N} v^{2}\right) \tilde{\sigma}+\frac{1}{2} v^{2} G_{\pi}^{-1}[0, \chi] \\
& \left.-\frac{N}{8 \lambda} \tilde{\chi}^{2}-\frac{\sqrt{2}}{2} \chi v^{2}+\frac{\lambda}{N} v^{4}-\epsilon v\right],
\end{aligned}
$$

where we have introduced the function

$$
G_{\pi}^{-1}[q, \chi] \equiv q^{2}-2 \bar{\mu}^{2}+\sqrt{2} \chi .
$$

Now comes our approximation for the auxiliary field. $\chi$ is not going to be integrated out, but treated at mean-field level, so that it contains no fluctuations. In particular, we apply this simplification for the quadratic term in the action. At mean field (note that we will keep the same notation $\chi$, instead of using $\langle\chi\rangle$ as they coincide in this approximation),

$$
\tilde{\chi}=\chi-2 \sqrt{2} \frac{\lambda}{N} v^{2}
$$

So the quadratic term reads

$$
-\frac{N}{8 \lambda} \tilde{\chi}^{2}=-\frac{N}{8 \lambda} \chi^{2}+\frac{\sqrt{2}}{2} v^{2} \chi-\frac{\lambda}{N} v^{4} .
$$

The last two factors are cancelled in the action (20). We are going to trade $\chi$ by $G_{\pi}^{-1}[0, \chi]$. Using Eq. (22), the quadratic terms is

$$
-\frac{N}{8 \lambda} \chi^{2}=-\frac{N}{16 \lambda}\left(G_{\pi}^{-1}[0, \chi]\right)^{2}-\frac{N}{4 \lambda} \bar{\mu}^{2} G_{\pi}^{-1}-\frac{N}{4 \lambda} \bar{\mu}^{4} .
$$

We will neglect the last term in the action because it is a constant. Finally, we use the relations

$$
\frac{N \bar{\mu}^{2}}{4 \lambda}=\frac{f_{\pi}^{2}(\epsilon=0)}{2}=\frac{f_{\pi}^{2}}{2 \alpha^{2}}=\frac{N F^{2}}{2 \alpha},
$$

to introduce $F=v(T=0)$, i.e., the VEV at zero temperature.
The final action becomes

$$
\begin{aligned}
S\left[\pi^{a}, \boldsymbol{v}, \tilde{\sigma}, G_{\pi}^{-1}[0, \chi]\right]= & \int d^{4} x\left[\frac{1}{2} \pi^{a}\left(-\square_{E}+G_{\pi}^{-1}[0, \chi]\right) \pi^{a}\right. \\
& +\frac{1}{2} \tilde{\sigma}\left(-\square_{E}+G_{\pi}^{-1}[0, \chi]+8 \frac{\lambda}{N} v^{2}\right) \tilde{\sigma} \\
& +\frac{1}{2} v^{2} G_{\pi}^{-1}[0, \chi]-\frac{N F^{2}}{2 \alpha^{2}} G_{\pi}^{-1}[0, \chi] \\
& \left.-\frac{N}{16 \lambda}\left(G_{\pi}^{-1}[0, \chi]\right)^{2}-\epsilon v\right] .
\end{aligned}
$$

Notice that $G_{\pi}^{-1}[q, \chi]$ is nothing but the inverse of the pion propagator in Fourier space. The inverse propagator of the $\tilde{\sigma}$ field is

$$
G_{\tilde{\sigma}}^{-1}[q, \chi]=G_{\pi}^{-1}[q, \chi]+8 \frac{\lambda}{N} v^{2} .
$$

In order to generate the effective potential for $v$, we now integrate out the fluctuations. By performing a standard Gaussian integration of the pions, we get

$$
\begin{gathered}
\int \mathcal{D} \pi^{a} \exp \left(-\int d^{4} x \frac{1}{2} \pi^{a}\left[-\square_{E}+G_{\pi}^{-1}[0, \chi]\right] \pi^{a}\right) \\
=\int d^{4} x \exp \left(\frac{N}{2} \int T \sum_{n \in Z} \frac{d^{3} q}{(2 \pi)^{3}} \log G_{\pi}^{-1}[q, \chi]\right),
\end{gathered}
$$

where $q_{0}=2 \pi n T$ is the well-known Matsubara frequency appearing in finite temperature computations. The same procedure can be applied to integrate out $\tilde{\sigma}$. To be able to perform the integrations, we have assumed that the auxiliary field (or $G_{\pi}^{-1}[0, \chi]$ ) is homogeneous, i.e., it does not depend on $x$. This assumption is also taken for the VEV of the $\sigma$ field and allows us to obtain a simple representation in Fourier space, where different modes do not mix between them and the integration is straightforward.

The effective potential (density) reads

$$
\begin{aligned}
V_{\mathrm{eff}}\left(v, G_{\pi}^{-1}[0, \chi]\right)= & \frac{1}{2}\left(v^{2}-\frac{N F^{2}}{\alpha^{2}}\right) G_{\pi}^{-1}[0, \chi] \\
& -\frac{N}{16 \lambda}\left(G_{\pi}^{-1}[0, \chi]\right)^{2}-\epsilon v \\
& +\frac{N}{2} \Psi_{\boldsymbol{\beta}} \log G_{\pi}^{-1}[q, \chi] \\
& +\frac{1}{2} \int_{\boldsymbol{\beta}} \log G_{\tilde{\sigma}}^{-1}[q, \chi],
\end{aligned}
$$

with

$$
\mathcal{f}_{\boldsymbol{\beta}}=T \sum_{n \in Z} \int \frac{d^{3} q}{(2 \pi)^{3}}
$$

Looking at the $N$ power counting of the different terms in the effective potential, one finds that all of them behave as $\mathcal{O}(N)$ except the last one. Therefore, the contribution of the Higgs to the effective potential is suppressed in the large- $N$ limit by one power of $N$, and it will be neglected in the following. Then, the effective potential becomes 

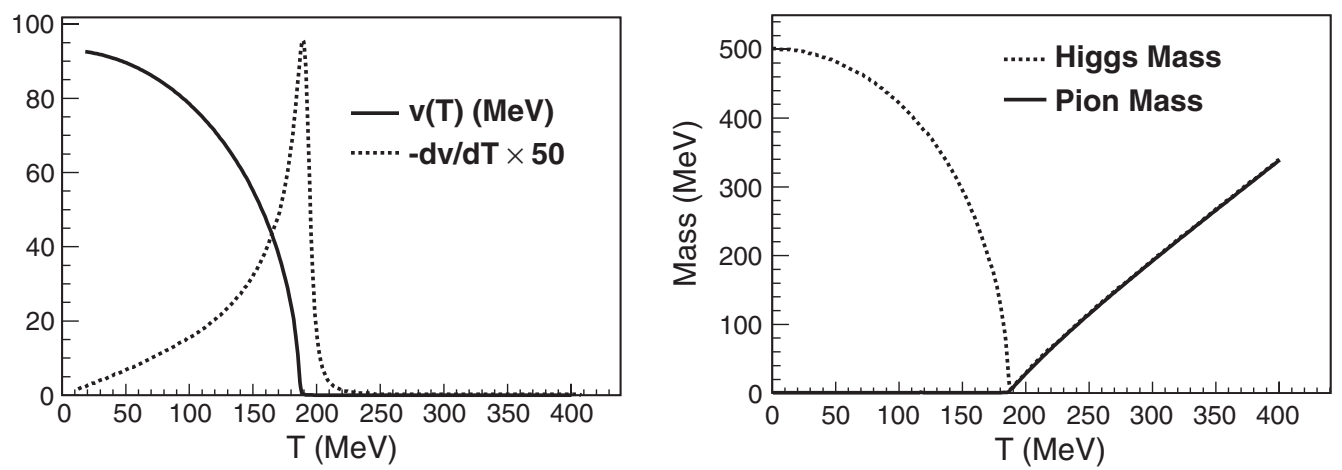

FIG. 1. Second-order phase transition. Left panel: Order parameter or VEV for $\tilde{\sigma}$ and its susceptibility. Right panel: Higgs and pion effective masses.

$$
\begin{aligned}
V_{\mathrm{eff}}\left(v, G_{\pi}^{-1}[0, \chi]\right)= & \frac{1}{2}\left(v^{2}-\frac{N F^{2}}{\alpha^{2}}\right) G_{\pi}^{-1}[0, \chi] \\
& -\frac{N}{16 \lambda}\left(G_{\pi}^{-1}[0, \chi]\right)^{2}-\epsilon v \\
& +\frac{N}{2} \Psi_{\beta} \log G_{\pi}^{-1}[q, \chi] .
\end{aligned}
$$

The last term needs to be regulated because it contains a divergence. This divergence can be absorbed by a proper renormalization of the quartic coupling [9]. Thus, the renormalized ( $\mu$-independent) effective potential finally reads

$$
\begin{aligned}
V_{\mathrm{eff}}\left(v, G^{-1}[0, \chi]\right)= & \frac{1}{2}\left(v^{2}-\frac{N F^{2}}{\alpha^{2}}\right) G_{\pi}^{-1}[0, \chi]-\epsilon v \\
& -\frac{N}{2} g_{0}\left(T, G_{\pi}^{-1}[0, \chi]\right) \\
& -\frac{N}{16}\left(G_{\pi}^{-1}[0, \chi]\right)^{2}\left[\frac{1}{\lambda_{R}(\mu)}\right. \\
& \left.-\frac{1}{4 \pi^{2}} \log \left(\frac{\sqrt{e} G_{\pi}^{-1}[0, \chi]}{\mu^{2}}\right)\right],
\end{aligned}
$$

where the function $g_{0}\left(T, G_{\pi}^{-1}[0, \chi]\right)$ is defined in Appendix A.
The stationary conditions are given by

$$
\left.\frac{d V_{\mathrm{eff}}}{d G_{\pi}^{-1}[0, \chi]}\right|_{G_{\pi}^{-1}[0, \chi]=G_{\pi, 0}^{-1}[0, \chi]}=0,\left.\quad \frac{d V_{\mathrm{eff}}}{d v}\right|_{v=v_{0}}=0
$$

and they provide $G_{\pi, 0}^{-1}[0, \chi]$ and the order parameter $v_{0}$ in this approximation. The effective mass of the pion is obtained as $m_{\pi}^{2}=G_{\pi, 0}^{-1}[0, \chi]$, and the effective Higgs mass as $M_{R}^{2}=G_{\pi, 0}^{-1}[0, \chi]+8 \frac{\lambda}{N} v_{0}^{2}$, which has the same form as Eq. (11).

For the $\epsilon=0$ case, there is no explicit symmetrybreaking term, and we expect to have a second-order phase transition defined by the critical temperature $T_{c}=$ $\sqrt{12} F=\sqrt{12 / N} f_{\pi}$. To obtain the numerical results appearing in Fig. 1, we have used a vanishing pion mass at $T=0$ (with $N=3$ ), a Higgs mass of $M_{R}=500 \mathrm{MeV}$, and $v(T=0)=93 \mathrm{MeV}$. In the left panel, we show the behavior of $v(T)$ which follows the analytical solution $v(T)=f_{\pi} \sqrt{1-T^{2} / T_{c}^{2}}$. The numerical critical temperature coincides with the theoretical value of $T_{c}=2 f_{\pi}=$ $186 \mathrm{MeV}$. We also show the susceptibility defined as minus the $T$ derivative of the order parameter. Its peak shows the position of the critical temperature. In the right panel, we show the effective masses as a function of the temperature. The effective mass of the pions at $T<T_{c}$ must be

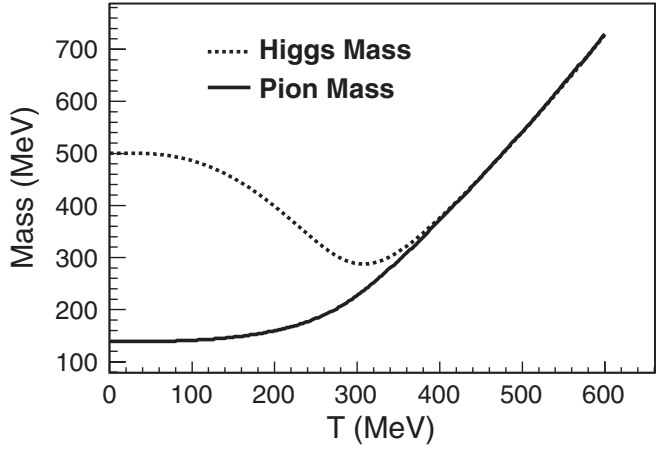

FIG. 2. Same as Fig. 1 but in the crossover case, when the pion mass is fixed at $T=0$ to $m_{\pi}=138 \mathrm{MeV}$. 
always zero according to the Goldstone theorem (numerically, it is fixed at $0.5 \mathrm{MeV}$ at $T=0$ in order to avoid computational problems). At $T_{c}$, it starts growing with temperature in the symmetric phase. The effective mass of the Higgs follows the same pattern as the order parameter becoming zero at $T_{c}$. For higher temperatures, it increases until being degenerate with the effective pion mass as expected because of the restoration of the $S O(N+1)$ symmetry.

For the $\epsilon \neq 0$ case, a crossover instead of a real phase transition is expected (this is the same situation as adding an external magnetic field to a ferromagnet). We fix $m_{\pi}=$ $138 \mathrm{MeV}$ at zero temperature and the same values for $M_{R}$ and $f_{\pi}$ taken for the $\epsilon=0$ case. The results are shown in Fig. 2. The left panel shows the order parameter $v(T)$ which decreases with temperature and never becomes exactly zero. The crossover temperature can be defined as the position of the peak in the susceptibility which we plot in dotted line. In the right panel, we show the pion thermal mass (solid line) and the Higgs mass (dotted line). They are degenerate for high temperatures.

\section{SHEAR VISCOSITY OVER ENTROPY DENSITY}

The shear viscosity for the $\mathrm{L} \sigma \mathrm{M}$ was obtained in Ref. [9]. In the following, we will briefly review the method used there with some minimal changes. The transport equation for the one-particle distribution function $f_{p}(t, \mathbf{x})$ is

$$
\frac{d f_{p}(t, \mathbf{x})}{d t}=C\left[f_{p}\right]
$$

Taking into account only elastic collisions (we will discuss later the influence of inelastic terms), this equation reads

$$
\begin{aligned}
\frac{\partial f_{p}(t, \mathbf{x})}{\partial t}+\frac{\mathbf{p}}{E_{p}} \cdot \nabla f_{p}(t, \mathbf{x})= & \frac{N}{2} \int d \Gamma_{12,3 p}\left[f_{1} f_{2}\left(1+f_{3}\right)\right. \\
& \times\left(1+f_{p}\right)-f_{3} f_{p}\left(1+f_{1}\right) \\
& \left.\times\left(1+f_{2}\right)\right],
\end{aligned}
$$

where

$d \Gamma_{12,3 p}=\frac{1}{2 E_{p}} \prod_{i=1}^{3} \frac{d \mathbf{k}_{i}}{(2 \pi)^{3} 2 E_{i}} \overline{|T|^{2}}(2 \pi)^{4} \delta^{(4)}\left(k_{1}+k_{2}-k_{3}-p\right)$.

At first order in the Chapman-Enskog expansion, the distribution function is expressed as the local equilibrium distribution function plus a small correction:

$$
f_{p}=n_{p}+f_{p}^{(1)},
$$

where $n_{p}$ is the local Bose-Einstein distribution function

$$
n_{p}(t, \mathbf{x})=\frac{1}{e^{\frac{p^{\alpha} \alpha_{\alpha}(t, \mathbf{x})-\mu(t, \mathbf{x})}{T(t, \mathbf{x})}}-1} .
$$

The Boltzmann-Uehling-Uhlenbeck (BUU) equation is then linearized in $f_{p}^{(1)}$ :

$$
\begin{aligned}
E_{p} & \frac{\partial n_{p}(t, \mathbf{x})}{\partial t}+\mathbf{p} \cdot \nabla n_{p}(t, \mathbf{x}) \\
= & -\frac{N E_{p}}{2} \int d \Gamma_{12,3 p}\left(1+n_{1}\right)\left(1+n_{2}\right) n_{3} n_{p}\left[\frac{f_{3}^{(1)}}{n_{3}\left(1+n_{3}\right)}\right. \\
& \left.+\frac{f_{p}^{(1)}}{n_{p}\left(1+n_{p}\right)}-\frac{f_{1}^{(1)}}{n_{1}\left(1+n_{1}\right)}-\frac{f_{2}^{(1)}}{n_{2}\left(1+n_{2}\right)}\right]
\end{aligned}
$$

The left-hand side depends only on the space-time derivatives of $n_{p}$, which can be explicitly obtained using the Euler and continuity equations.

The shear viscosity can be also expressed in terms of $f_{p}^{(1)}$ :

$$
2 \eta \tilde{V}_{i j}=N \int \frac{d^{3} p}{\left(2 \pi^{3}\right) E_{p}} f_{p}^{(1)} p_{i} p_{j},
$$

where $\tilde{V}_{i j}=\partial_{i} V_{j}+\partial_{j} V_{i}-\frac{1}{3} \delta_{i j} \nabla \cdot \mathbf{V}$ is the shear gradient of the velocity field.

The left-hand side of the BUU equation also carries the same gradient (neglecting the influence of other transport coefficients),

$E_{p} \frac{\partial n_{p}(t, \mathbf{x})}{\partial t}+\mathbf{p} \cdot \nabla n_{p}(t, \mathbf{x})=\beta n_{p}\left(1+n_{p}\right) p^{i} p^{j} \tilde{V}_{i j}$.

In order to cancel out this factor from the BUU equation and also from Eq. (41), the function $f_{p}^{(1)}$ is taken as

$$
f_{p}^{(1)}=-n_{p}\left(1+n_{p}\right) \beta^{3} B(p) p^{i} p^{j} \tilde{V}_{i j},
$$

with $B(p)$ being an unknown function of $p$. Inserting Eq. (43) into Eq. (41), one gets

$$
\eta=\frac{N}{30 \pi^{2} T^{3}} \int d p \frac{p^{6}}{E_{p}} n_{p}\left(1+n_{p}\right) B(p) .
$$

It is convenient to write this formula in terms of the adimensional variables

$$
x=\frac{E_{p}}{m_{\pi}}, \quad y=\frac{m_{\pi}}{T} .
$$

Introducing the integration measure $d \mu_{\eta}(x ; y)$ defined in Appendix B, we find

$$
\eta=\frac{N m_{\pi}^{6}}{30 \pi^{2} T^{3}} \int_{\Omega} d \mu_{\eta}(x ; y) B(x) .
$$

This $B(x)$ function can be expanded in terms of the polynomial basis $P_{n}(x)$ defined in Appendix B:

$$
B(x)=\sum_{n=0}^{\infty} b_{n} P_{n}(x) .
$$

By projecting the BUU equation into the space generated by $P_{n}$, one gets 


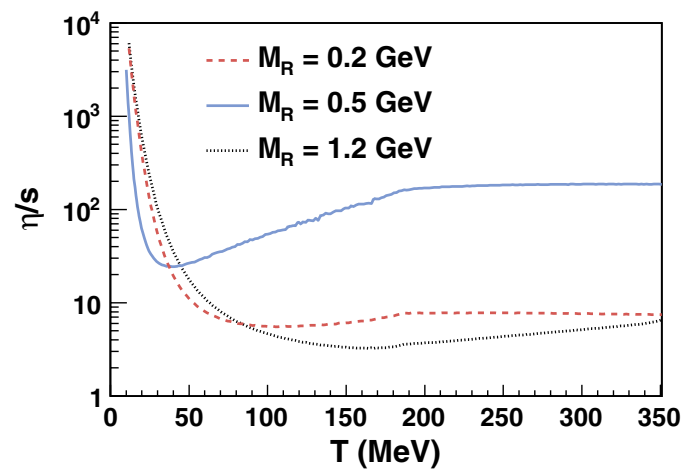

FIG. 3 (color online). Viscosity over entropy density in the $\mathrm{L} \sigma \mathrm{M}$ at large $N$ for different values of $M_{R}$.

$$
K^{0} \delta_{l 0}=\sum_{n=0}^{N} b_{n} \mathcal{C}_{n l}^{\eta}
$$

where the functions $K^{i}$ are also defined in Appendix B. The collision integrals read

$$
\begin{aligned}
\mathcal{C}_{n l}^{\eta}= & \frac{N \pi^{2}}{4 m_{\pi}^{2} T^{2}} \int \prod_{m=1}^{4} \frac{d^{3} k_{m}}{(2 \pi)^{3} 2 E_{m}} \overline{|T|^{2}}(2 \pi)^{4} \\
& \times \delta^{(4)}\left(k_{1}+k_{2}-k_{3}-p\right)\left(1+n_{1}\right)\left(1+n_{2}\right) n_{3} n_{p} \\
& \times\left[\frac{p_{i} p_{j}}{m_{\pi}^{2}} P_{l}(p)+\frac{k_{3 i} k_{3 j}}{m_{\pi}^{2}} P_{l}\left(k_{3}\right)-\frac{k_{1 i} k_{1 j}}{m_{\pi}^{2}} P_{l}\left(K_{1}\right)\right. \\
& \left.-\frac{k_{2 i} k_{2 j}}{m_{\pi}^{2}} P_{l}\left(k_{2}\right)\right]\left[\frac{p^{i} p^{j}}{m_{\pi}^{2}} P_{n}(p)+\frac{k^{3 i} k^{3 j}}{m_{\pi}^{2}} P_{n}\left(k_{3}\right)\right. \\
& \left.-\frac{k^{1 i} k^{1 j}}{m_{\pi}^{2}} P_{n}\left(k_{1}\right)-\frac{k^{2 i} k^{2 j}}{m_{\pi}^{2}} P_{n}\left(k_{2}\right)\right] .
\end{aligned}
$$

At the lowest order in the expansion (47), the shear viscosity can be written as

$$
\eta=\frac{N m_{\pi}^{6}}{30 \pi^{2} T^{3}} \frac{K_{0}^{2}}{\mathcal{C}_{00}^{\eta}},
$$

where the pion effective mass $m_{\pi}$ is now taken from the gap equations of the effective potential and

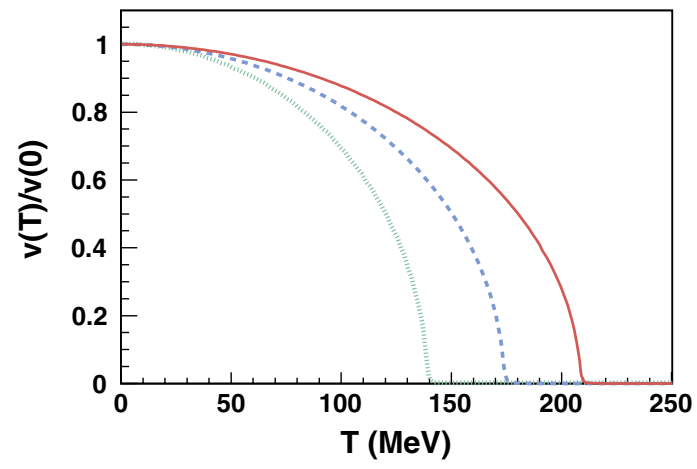

$$
\begin{aligned}
\mathcal{C}_{00}^{\eta}= & \frac{N \pi^{2}}{4 m_{\pi}^{2} T^{2}} \int \prod_{m=1}^{4} \frac{d^{3} k_{m}}{(2 \pi)^{3} 2 E_{m}} \overline{|T|^{2}}(2 \pi)^{4} \\
& \times \delta^{(4)}\left(k_{1}+k_{2}-k_{3}-p\right)\left(1+n_{1}\right)\left(1+n_{2}\right) n_{3} n_{p} \\
& \times\left[\frac{p_{i} p_{j}}{m_{\pi}^{2}}+\frac{k_{3 i} k_{3 j}}{m_{\pi}^{2}}-\frac{k_{1 i} k_{1 j}}{m_{\pi}^{2}}-\frac{k_{2 i} k_{2 j}}{m_{\pi}^{2}}\right] \\
& \times\left[\frac{p^{i} p^{j}}{m_{\pi}^{2}}+\frac{k^{3 i} k^{3 j}}{m_{\pi}^{2}}-\frac{k^{1 i} k^{1 j}}{m_{\pi}^{2}}-\frac{k^{2 i} k^{2 j}}{m_{\pi}^{2}}\right] .
\end{aligned}
$$

The scattering amplitude in the large- $N$ limit has been extensively described in Ref. [9], which includes the treelevel $\pi-\pi$ amplitude and its resummation in pion loops (as they are of the same order in $N$ ). The finite pion mass gives rise to new couplings to the pion scattering [32], and the corresponding amplitude should be added to the chiral one. In the large- $N$ limit, the $s$-channel scattering is the leading one. As the amplitude is $\mathcal{O}(1 / N)$, the total cross section is $\mathcal{O}(1 / N)$, but the average cross section (which is included in the collision integral) is $\mathcal{O}\left(1 / N^{2}\right)$.

Note that, as $\mathcal{C}_{n l}^{\eta} \sim \mathcal{O}(1 / N)$, the shear viscosity is $\mathcal{O}\left(N^{2}\right)$. This is expected [33] since the shear viscosity is proportional to the inverse coupling constant squared, and this is suppressed by one power of $N$.

The obtained numerical results for $N=3, m_{\pi}(T=0)=0$ and Higgs masses $M_{R}=0.2,0.5$ and $1.2 \mathrm{GeV}$ are shown in Fig. 3. They are similar to that appearing in our Ref. [9] where we have used a slightly different parametrization for $f_{p}^{(1)}$. We have found a minimum of $\eta / s$ for the three cases, always greater than the KSS bound $1 /(4 \pi)$. However, the exact position of the minimum depends on the value of $M_{R}$.

To check whether the minimum of $\eta / s$ corresponds to the location of the critical temperature, one must compare the previous plot with the order parameter. We show this in Fig. 4 at different values of $F(T=0)$. In the left panel, we show the normalized value of $v(T)$. The position of the critical temperature (where the order parameter first vanishes) depends linearly on $F$. The same behavior is followed by the minimum viscosity over entropy density

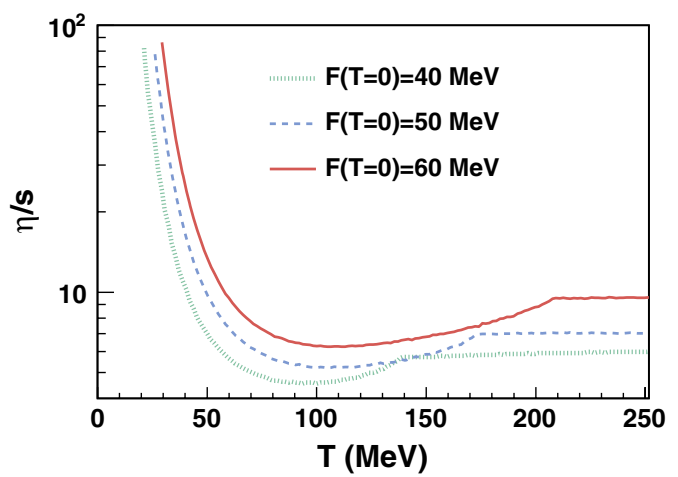

FIG. 4 (color online). Comparison between the minimum of the viscosity over entropy density and the position of the critical temperature. The minimum is located close to it but slightly below. 
shown in the right panel. The position of this minimum is close to $T_{c}$, but not exactly there (as shown in Fig. 4) but slightly below. Notice that $T_{c}$ is $M_{R}$-independent in the large- $N$ approximation considered here.

\section{BULK VISCOSITY OVER ENTROPY DENSITY}

In this section, we will perform the calculation of the bulk viscosity in the $\mathrm{L} \sigma \mathrm{M}$ in the large- $N$ approximation. We start by considering both elastic and inelastic scatterings. Therefore, we do not introduce a pion chemical potential in the calculation, as the pion number is not conserved in principle. This fact simplifies the thermodynamics with respect to our previous work [15] since, instead of using the isochoric speed of sound $v_{n}$ and the compressibility $\kappa_{\epsilon}^{-1}$, one only needs to consider the adiabatic speed of sound,

$$
v_{S}^{2}=\left(\frac{\partial P}{\partial \epsilon}\right)_{S / n},
$$

where $P$ is the pressure and $\epsilon$ the energy density.

Additionally, as we will use a quasiparticle description of the scalar fields, we must introduce a nonvanishing term $d m_{\pi} / d T$ which enters in the left-hand side of the BUU equation:

$$
\begin{aligned}
& \left.p_{\mu} \partial^{\mu} n_{p}(x)\right|_{\zeta} \\
& =\beta n_{p}\left(1+n_{p}\right)\left(\frac{p^{2}}{3}-E_{p}^{2} v_{S}^{2}+T m_{\pi} \frac{d m_{\pi}}{d T} v_{S}^{2}\right) \nabla \cdot \mathbf{V} .
\end{aligned}
$$

Here, it is useful to define a new $T$-dependent parameter $\tilde{m}$ which includes the derivative of the thermal mass:

$$
\tilde{m}^{2} \equiv m_{\pi}^{2}-T^{2} \frac{d m_{\pi}^{2}}{d T^{2}} .
$$

Then, the left-hand side reads

$$
\left.p_{\mu} \partial^{\mu} n_{p}(x)\right|_{\zeta}=\beta n_{p}\left(1+n_{p}\right)\left[\frac{p^{2}}{3}-v_{S}^{2}\left(p^{2}+\tilde{m}^{2}\right)\right] \nabla \cdot \mathbf{V} \text {. }
$$

The first-order Chapman-Enskog correction to the distribution function $f_{p}=n_{p}+f_{p}^{(1)}$ is

$$
f_{p}^{(1)}=-n_{p}\left(1+n_{p}\right) \beta A(p) \nabla \cdot \mathbf{V} .
$$

The linearized BUU equation reads

$$
\begin{aligned}
n_{p}\left(1+n_{p}\right)\left[\frac{p^{2}}{3}-v_{S}^{2}\left(p^{2}+\tilde{m}^{2}\right)\right] \\
=\mathcal{C}_{\mathrm{el}}+\mathcal{C}_{\mathrm{in}} \\
=\frac{N E_{p}}{2} \int d \Gamma_{12,3 p}\left(1+n_{1}\right)\left(1+n_{2}\right) n_{3} n_{p} \\
\quad \times\left[A(p)+A\left(k_{3}\right)-A\left(k_{1}\right)-A\left(k_{2}\right)\right]+\mathcal{C}_{\mathrm{in}},
\end{aligned}
$$

where we represent by $\mathcal{C}_{\mathrm{el}}$ the elastic collision operator and by $\mathcal{C}_{\text {in }}$ the part of the collision operator including inelastic scattering (that we do not explicitly detail here). As we have not fixed the pion chemical potential, this term should be present in order to allow particle number changing processes.

The stress-energy tensor $\tau^{\mu \nu}$ in presence of a thermal mass is written as [23,26,34]

$$
\tau^{\mu \nu}=N \int \frac{d^{3} p}{(2 \pi)^{3} E_{p}}\left(p^{\mu} p^{\nu}-u^{\mu} u^{\nu} T^{2} \frac{d m^{2}}{d T^{2}}\right) f_{p}^{(1)},
$$

which reduces to the usual stress-energy tensor when the mass is $T$-independent.

This new term does not modify the expression for the bulk viscosity in terms of $f_{p}^{(1)}$ because the former only depends on the spatial components of the stress-energy tensor, and in the local rest frame, one has $u^{i}=0$. Therefore, we have

$$
\zeta=\frac{N}{T} \int \frac{d^{3} p}{(2 \pi)^{3} E_{p}} n_{p}\left(1+n_{p}\right) A(p) \frac{p^{2}}{3} .
$$

However, this new term in the stress-energy tensor does change the form of the Landau-Lifschitz condition [35] $\tau^{00}=0$ (notice that this is the only condition, since the one fixing the particle density number out of equilibrium does not apply here):

$$
\tau^{00}=N \int \frac{d^{3} p}{(2 \pi)^{3} E_{p}}\left(p^{2}+\tilde{m}^{2}\right) f_{p}^{(1)}=0,
$$

As usual, this condition can be used to add a vanishing contribution to the bulk viscosity, making much easier the comparison with the BUU equation left-hand side (55),

$$
\zeta=\frac{N}{T} \int \frac{d^{3} p}{(2 \pi)^{3} E_{p}} n_{p}\left(1+n_{p}\right) A(p)\left[\frac{p^{2}}{3}-v_{S}^{2}\left(p^{2}+\tilde{m}^{2}\right)\right] .
$$

Next, we consider again the same adimensional variables we have used for the shear viscosity $\left(x=E_{p} / m_{\pi}, y=\right.$ $\left.m_{\pi} / T\right)$ and also an integration measure $d \mu_{\zeta}$. This integration measure is described in detail in Appendix A including its corresponding scalar product, the norm, the moments, the functions $I^{i}$, and the polynomial basis. The bulk viscosity is then expressed as the following scalar product:

$$
\zeta=\frac{N m_{\pi}^{4}}{2 \pi^{2} T}\left\langle A(x) \mid P_{2}(x)\right\rangle_{\zeta}
$$

The projected BUU equation is obtained by multiplying both sides of Eq. (57) by $\frac{1}{4 \pi m_{\pi}^{4} E_{p}} P_{l}(x) d^{3} p$ and integrating over the three-momentum:

$$
\begin{aligned}
& \left\langle P_{l}(x) \mid P_{2}(x)\right\rangle \\
& =\frac{N}{8 \pi m_{\pi}^{4}} \int d^{3} p \int d \Gamma_{12,3 p}\left(1+n_{1}\right)\left(1+n_{2}\right) n_{3} n_{p} \\
& \quad \times P_{l}(x)\left[A(p)+A\left(p_{3}\right)-A\left(p_{1}\right)-A\left(p_{2}\right)\right]+\left\langle\mathcal{C}_{\text {in }}\right\rangle .
\end{aligned}
$$


There is one important remark concerning the solution of the linearized BUU. In this linearized equation, only one zero mode is present. When $A(p)$ is proportional to $x$, the right-hand side is zero due to the energy-conservation law. However, due to the presence of the inelastic collision operator, the zero mode associated to the particle conservation $(A(p) \propto 1)$ is absent.

In the left-hand side, it is easy to check that whereas $\left\langle P_{1} \mid P_{2}\right\rangle=0$, we have $\left\langle P_{0} \mid P_{2}\right\rangle \neq 0$, which is consistent with the previous remark. The BUU equation is solvable in the entire Hilbert space of solutions [36].

Due to arguments of final phase-space and suppression in the large- $N$ limit (which will be detailed later), we will not consider the inelastic processes in the collision integral and retain only the $2 \rightarrow 2$ processes. This simplification causes an inconsistency in the BUU equation, as the projection of the BUU onto $P_{0}$ gives different results on both sides of the equation. This inconsistency is avoided by solving the equation in the subspace perpendicular to the "accidental" zero mode $P_{0}=1$.

Therefore, we expand the $A(x)$ solution:

$$
A(x)=\sum_{n=1}^{\infty} a_{n} P_{n}(x)
$$

After symmetrization of the collision integral, the BUU equation finally reads

$$
\delta_{l 2}\left\|P_{2}(x)\right\|^{2}=\sum_{n=1}^{\infty} a_{n} \mathcal{C}_{n l}
$$

with

$$
\begin{aligned}
\mathcal{C}_{n l}= & \frac{N \pi^{2}}{4 m_{\pi}^{4}} \int \prod_{i=1}^{4} \frac{d^{3} k_{i}}{(2 \pi)^{3} 2 E_{i}} \overline{|T|^{2}}(2 \pi)^{4} \\
& \times \delta^{(4)}\left(k_{1}+k_{2}-k_{3}-p\right)\left(1+n_{1}\right) \\
& \times\left(1+n_{2}\right) n_{3} n_{p} \Delta\left[P_{n}(x)\right] \Delta\left[P_{l}(x)\right],
\end{aligned}
$$

where $\Delta\left[P_{n}(x)\right] \equiv P_{n}(x)+P_{n}\left(x_{3}\right)-P_{n}\left(x_{1}\right)-P_{n}\left(x_{2}\right)$.

For $l=1$, one gets the identity $0=0$, which does not determine the coefficient $a_{1}$. The first nontrivial case corresponds to $l=2$, for which the solution of the BUU equation reads

$$
a_{2}=\frac{\left\|P_{2}(x)\right\|^{2}}{\mathcal{C}_{22}} .
$$

By introducing it in the formula for the bulk viscosity, one finds

$$
\zeta=\frac{N m_{\pi}^{4}}{2 \pi^{2} T} a_{2}\left\|P_{2}(x)\right\|^{2}=\frac{N m_{\pi}^{4}}{2 \pi^{2} T}\left(\frac{1}{3}-v_{S}^{2}\right) \frac{1}{\mathcal{C}_{22}}\left(I_{4}-\frac{I_{2} I_{3}}{I_{1}}\right) .
$$

In the following, we proceed to the discussion of the results for the bulk viscosity over entropy density. They will depend on the value of the pion mass at zero temperature, i.e., on having a second-order phase transition or a crossover. We will begin with the latter.

\section{A. Crossover}

We start with the explicit symmetry-breaking case, for which we set a nonzero pion mass at $T=0$. In order to get numerical results, a possible choice could be the physical pion mass $m_{\pi}(T=0)=138 \mathrm{MeV}$. The effective mass depends on temperature in such a way that $m_{\pi}(T)>$ $m_{\pi}(T=0)=138 \mathrm{MeV}$. For this reason, and analogously to the physical pion gas in Ref. [15], the inelastic terms in the collision operator $(1 \rightarrow 3$ or $2 \rightarrow 4)$ are suppressed by the Boltzmann exponential factor in the final phase space $e^{-2 m_{\pi} / T}$ (the inverses being too improbable to occur if the gas is dilute).

However, the crossover case does not have a precise definition of the "critical" temperature. Here, we will define it as the point where the minus derivative of the order parameter (the susceptibility) peaks. From the left panel of Fig. 2, we obtain a value of $T_{\mathrm{cr}}=261 \mathrm{MeV}$.

In Fig. 5, we show the squared speed of sound for the pions together with the result from a pion gas with a constant (temperature-independent) mass of $m_{\pi}=$ $138 \mathrm{MeV}$. The difference between the two curves is attributed to the effective mass of the quasiparticles. It is important to remark here that both results correspond to the noninteracting gas, i.e., ideal gas. The introduction of interactions in the thermodynamic functions (for example, through the free energy obtained from the effective potential) would be inconsistent with the conception of the Chapman-Enskog expansion at first order.

The speed of sound turns out to be a monotonic function of the temperature. It takes the conformal value $v_{S}^{2}=1 / 3$ at $T=259 \mathrm{MeV}$, very close to the crossover temperature. By Eq. (B20), one can check that, at this precise point,

$$
\frac{d m_{\pi}}{d T}=\frac{m_{\pi}}{T}
$$

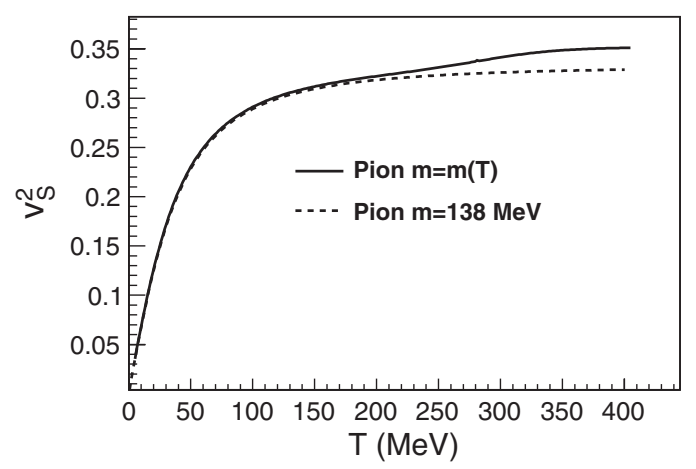

FIG. 5. $v_{S}^{2}$ in the L $\sigma \mathrm{M}$ at large $N$ for the crossover case (solid line) compared with the speed of sound of a pion mass with a constant mass of $138 \mathrm{MeV}$. 


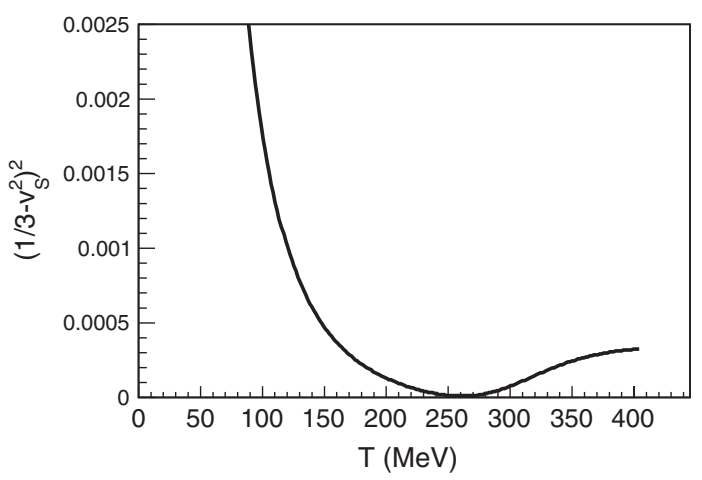

FIG. 6. $\left(1 / 3-v_{S}^{2}\right)^{2}$ as a measure of the violation of the conformality of the system. The bulk viscosity qualitatively follows this factor.

When $v_{S}^{2}=1 / 3$, the squared norm of the source $P_{2}$ identically vanishes. Therefore, the bulk viscosity is zero because of Eq. (68). More generally, the bulk viscosity features the factor $\left(\frac{1}{3}-v_{S}^{2}\right)^{2}$ which measures the violation of conformality in the system [23]. Thus, when the speed of sound takes values far from the conformal one, the bulk viscosity will be non-negligible, whereas if $v_{S}^{2}$ is near the conformal value [37], the bulk viscosity will be close to zero. We plot the factor $\left(\frac{1}{3}-v_{S}^{2}\right)^{2}$ in Fig. 6 .

Another measure of the loss of conformality is the socalled interaction measure. In $D=3+1$ dimensions, it is defined as $\langle\theta\rangle_{T}=\epsilon-3 P$, where $\epsilon$ is the energy density of the gas and $P$ its pressure. Using the relativistic equation of state $P+\epsilon=T s$, it can be written as $\langle\theta\rangle_{T}=T s-4 P$ as a function of the pressure and the entropy density. The interaction measure is shown in the left panel of Fig. 7. The result for the bulk viscosity over entropy density is plotted in the right panel of the same figure. As stated before, the qualitative shape of the $\zeta / s$ follows the behavior of $\left(1 / 3-v_{S}^{2}\right)^{2}$ shown in Fig. 6 .

A maximum in the bulk viscosity over entropy density is not seen at the crossover temperature. However, a minimum is found for $v_{S}^{2}=1 / 3$. Later, we will discuss the apparent contradiction with the claim of a maximum of $\zeta / \mathrm{s}$ appearing at the phase transition.

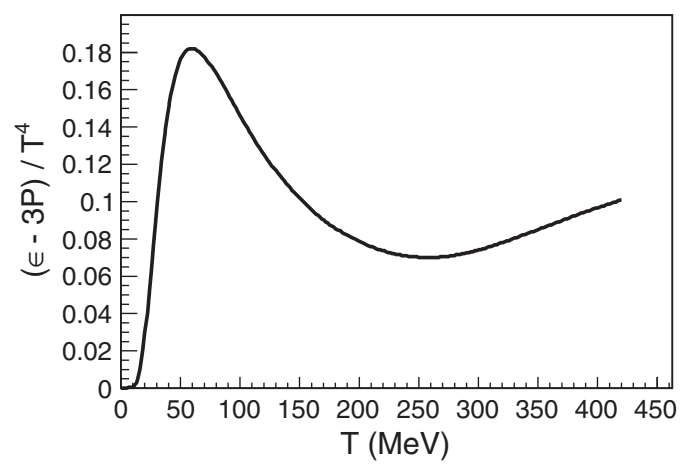

To end this section, we want to stress that this result is perfectly consistent (with due respect to the inevitable differences) with the result in Ref. [24] for the GrossNeveu model in the large- $N$ limit. In that case, no peak is seen in $\zeta / s$. More specifically, in the crossover case (which is the equivalent to this one) the speed of sound is monotonic and structureless like in our case. However, the speed of sound does not cross the conformal value because if $D=1+1$, then $\left.v_{S}\right|_{\text {CFT }}=1$, and this is the value to which the speed of sound approaches asymptotically at large temperatures [38]. Following our considerations, the bulk viscosity in that model should be a monotonic decreasing function on the temperature, going to zero at asymptotically higher temperatures (where the conformal value of the speed of sound is reached at $T \rightarrow \infty$ ).

In order to give a consistency check of the previous result, we will repeat the calculation in the chiral limit (at zero temperature) for pions.

\section{B. Second-order phase transition}

If the pion mass vanishes at $T=0$, then a second-order phase transition is expected. In this case, we can neglect the inelastic processes by a different argument. In the broken phase, as the pion mass vanishes, there is no Boltzmann suppression in the final phase space. However, in the large- $N$ limit, the tree-level amplitude for inelastic processes $(2 \rightarrow 4)$ carries one extra $1 / N$ factor. The average cross section (or the average scattering amplitude inside the collision operator) is order $1 / N$ for elastic scattering, but it is order $1 / N^{3}$ for the inelastic case, and therefore it is suppressed in the large- $N$ limit.

Before looking at $\zeta / s$, we show the speed of sound in Fig. 8. In the broken phase, the pions are massless, and the speed of sound takes the value of a conformal gas $v_{S}^{2}=1 / 3$ (note that the Higgs does not contribute because its effect is suppressed in the large- $N$ limit). When the critical temperature $T_{c}=186 \mathrm{MeV}$ is reached, the mass of pions starts to grow, and the speed of sound separates from the conformal value. In the right panel, we show the factor of loss of conformality $\left(1 / 3-v_{S}^{2}\right)^{2}$ which gives an idea of

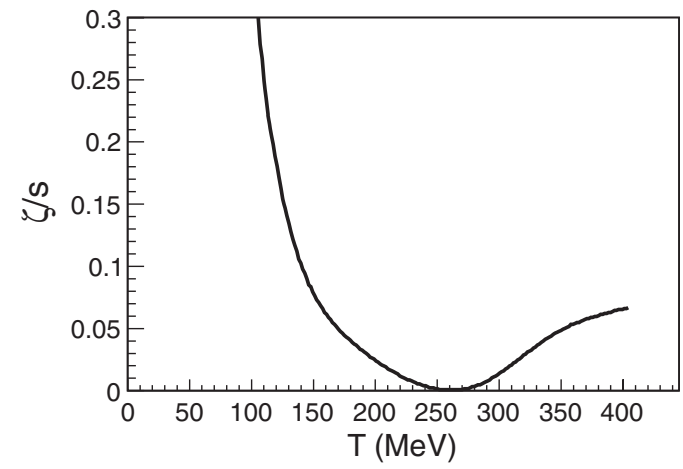

FIG. 7. Left panel: Interaction measure for the crossover case as a signal of loss of conformality in the system. Right panel: $\zeta / s$ in the $\mathrm{L} \sigma \mathrm{M}$ at large $N$ for the crossover case. 

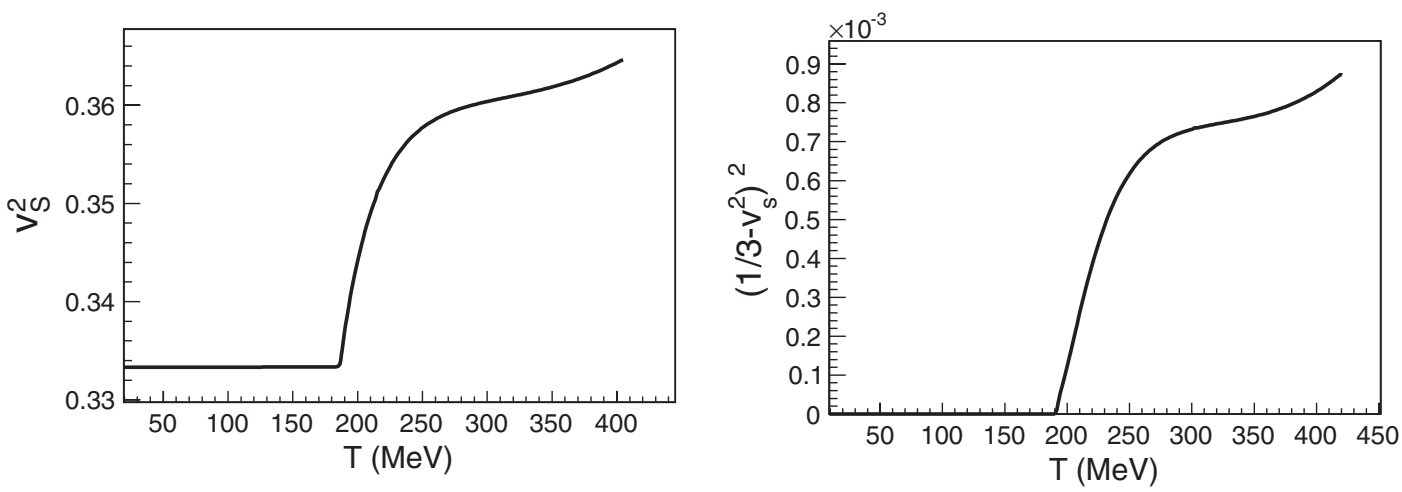

FIG. 8. Left panel: $v_{S}^{2}$ in the L $\sigma \mathrm{M}$ at large $N$ for the second-order phase transition case. Right panel: Factor $\left(1 / 3-v_{S}^{2}\right)^{2}$ which measures the violation of conformality.

the behavior of the bulk viscosity. Of course, we expect the bulk viscosity to vanish in the broken phase and to grow for $T>T_{c}$.

In the left panel of Fig. 9, the interaction measure is plotted. In the broken phase, it also vanishes as the gas is conformal. The $\zeta / s$ coefficient is also shown in Fig. 9. The bulk viscosity is zero in the broken phase, but it starts to grow and takes a monotonic behavior when temperature is increased.

Note that again this result is consistent with the GrossNeveu model at large $N$ in the chiral limit of fermion mass [24]. In the high temperature phase, the mass of the fermion field is exactly zero, and the speed of sound takes the conformal value of $v_{S}^{2}=1$. Therefore, the bulk viscosity turns out to be zero. No maximum is seen at the phase transition temperature. When decreasing the temperature (the fermion thermal mass increases), the bulk viscosity has a finite discontinuity and increases as $T \rightarrow 0$. This is exactly what happens in our case, but with the high- and low-temperature limits reversed.

Finally, we show how the second-order case connects with the crossover by slowly increasing the pion mass from $0.5 \mathrm{MeV}$ (our chiral value) up to $138 \mathrm{MeV}$. In Fig. 10, we plot the evolution of the pion and Higgs masses and the order parameter $v(T)$ as a function of the temperature and

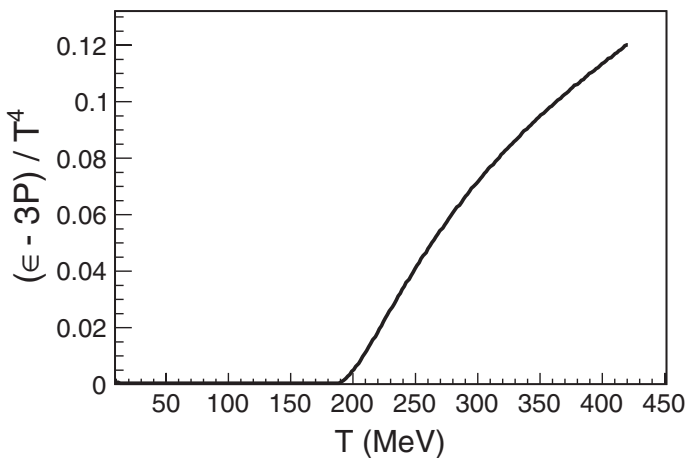

the pion mass at $T=0$. In Fig. 11, we show the squared speed of sound and the bulk viscosity.

\section{Discussion of the results}

In both cases, the second-order phase transition and the crossover, we have not obtained a maximum for the bulk viscosity at the phase transition. We have extensively studied the reasons for this fact. One of the keys to understand this result is the behavior of the speed of sound (or more generally, the equation of state). Conformality, or the lack of it, should be reflected in this factor either by taking the conformal value $v_{S}^{2}=1 / 3$ or going far from it. One can conclude that, in order to see a maximum of the bulk viscosity at the critical temperature, one should have a nonmonotonic behavior of $v_{S}^{2}$ near $T_{c}$. For instance, the bulk viscosity could approach the conformal value as $T \rightarrow T_{c^{-}}$, showing a sudden dip at $T_{c}$ and increasing again to the conformal value $1 / 3$ for $T>T_{c}$. This behavior would produce a maximum in the bulk viscosity. Actually, this is the scenario when the bulk viscosity is phenomenogically included in the QCD phase transition (see, for instance, Refs. [22,23,39]) and from the lattice QCD equation of state [40-42].

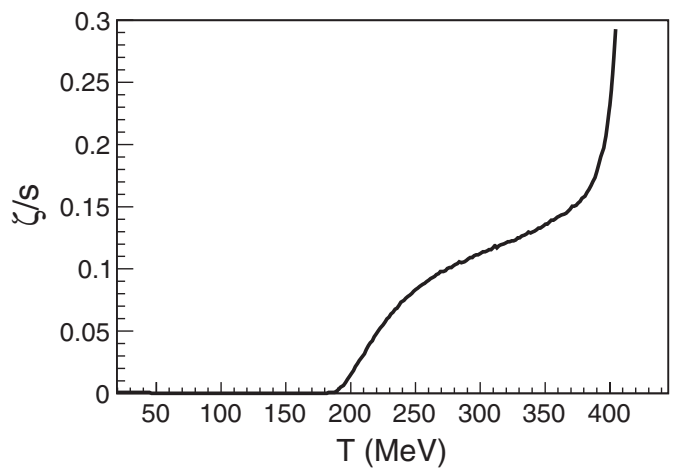

FIG. 9. Left panel: Interaction measure as a signature of loss of conformality of the system. Right panel: $\zeta / s$ in the L $\sigma \mathrm{M}$ at large $N$ for the second-order phase transition. 

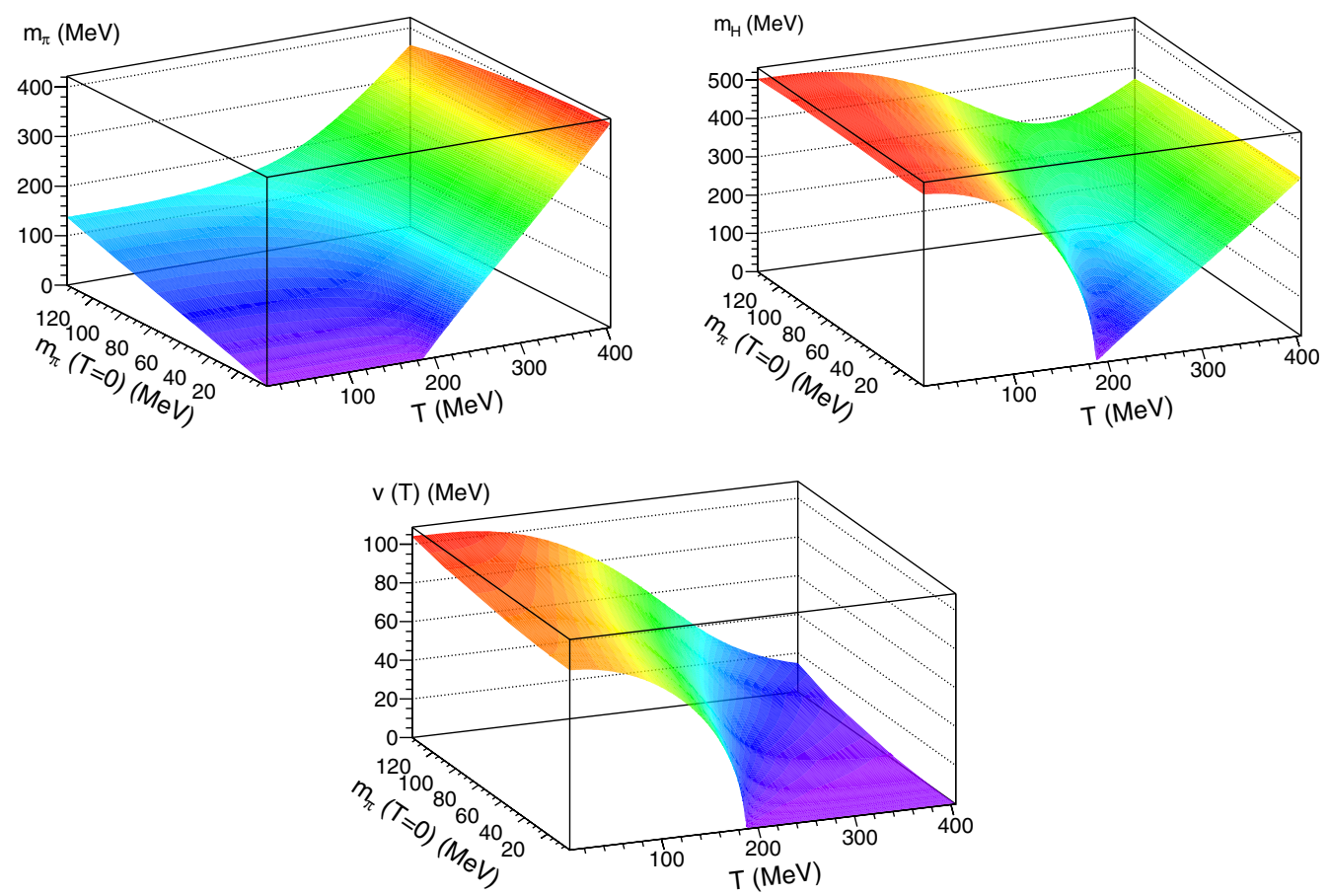

FIG. 10 (color online). Top panels: Pion and Higgs thermal masses. Bottom panel: Order parameter. All of them are plotted as a function of the temperature and the pion mass at $T=0$.

This behavior is also consistent with the second peak in the bulk viscosity showed in Ref. [14]. The nature of this peak can easily be understood from the speed-of-sound curve. In that work, a dilute gas of pions with a physical mass of $138 \mathrm{MeV}$ is considered. The speed of sound is obtained from finite temperature chiral perturbation theory at two loops and order $T^{8}$. In Fig. 12, we plot (dashed line) the results obtained by direct use of the formulas in Ref. [43] for the pressure of a pion gas. The local minimum of $v_{S}^{2}$ is responsible for the breaking of conformality around $T \sim 220 \mathrm{MeV}$, and, therefore, a maximum in the bulk viscosity is found in Ref. [14] at that temperature.

Focusing again in the $\mathrm{L} \sigma \mathrm{M}$ in the large- $N$ limit, the monotonic behavior of the speed of sound is inherited by the behavior of the thermal mass of the pion. The mild increase of the pion mass, without any indication of phase

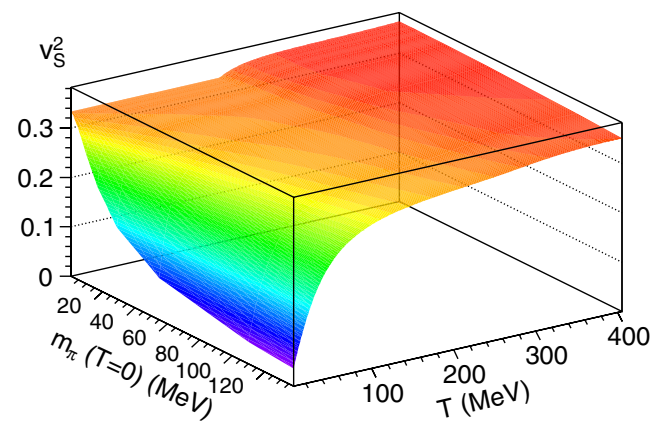

transition, determines the dependence on temperature of the speed of sound and eventually the bulk viscosity. This behavior is a consequence of the large- $N$ approximation, in which some of the interesting details of the model are washed out. In other words, the simplification of the large- $N$ limit in some aspects of the model is also accompanied by an oversimplification of the dynamics which eventually gives rise to the absence of a maximum in the bulk viscosity. In fact, as we have already mentioned, the dynamical renormalization group calculation is not expected to have a divergence of the bulk viscosity in the large- $N$ limit of this model [25]. This limit was also considered for the Gross-Neveu model in Ref. [24], and the results found there are perfectly compatible with ours.

The use of a more complicated dispersion relation or a different thermal effective mass behavior can notably

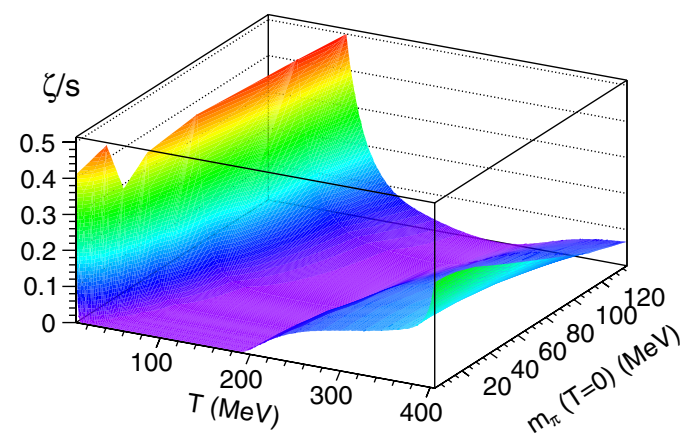

FIG. 11 (color online). Left panel: Speed of sound as a function of temperature and pion mass at zero temperature. Right panel: Bulk viscosity over entropy density. Note the nonstandard direction of the axes for the sake of clarity. 


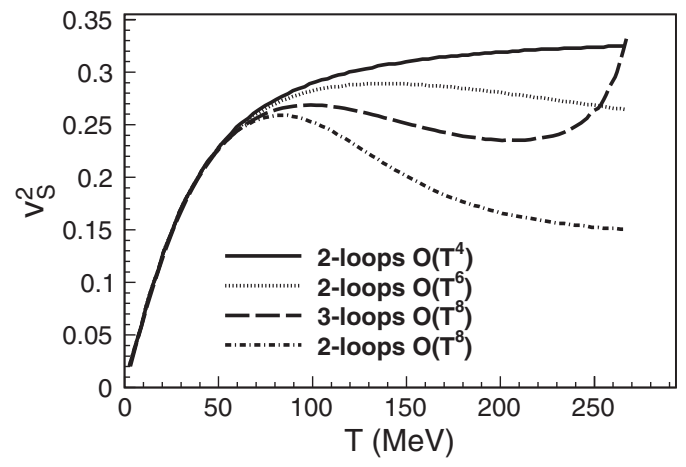

FIG. 12. Squared speed of sound of pions with physical mass of $138 \mathrm{MeV}$. We use thermal chiral perturbation theory [43] at different approximations.

change this result. A hint for this fact can be found in Ref. [23]. In that reference, the authors consider also the $\mathrm{L} \sigma \mathrm{M}$ model, but in a different way. First, they do not take the large- $N$ limit, and, therefore, the interactions among pions and Higgs must be included. However, in order to avoid some divergences in the cross section, they only consider constant scattering amplitudes (in fact, they do not take into account any correction to the amplitudes due to the finite pion mass). Second, they do not solve the BUU equation by the Chapman-Enskog expansion. Instead, they use the relaxation time approximation to obtain the bulk viscosity. Finally, the effective potential is calculated in the CJT approximation, which gives qualitative different results on the behavior of the effective masses and eventually on the speed of sound and bulk viscosity. In that reference, a peak in the bulk viscosity is found in the crossover case for some specific values of the Higgs masses.

Therefore, in order to conclude our study of the bulk viscosity in the $\mathrm{L} \sigma \mathrm{M}$, it seems to be interesting to consider a different effective mass to show that a maximum in the bulk viscosity is obtained without changing any other aspect of our calculation. Thus, we will use the Hartree approximation of the CJT theory as presented in Ref. [44] for the calculation of the order parameter and thermal masses. Doing so is not completely consistent since we are using the Hartree approximation for the effective potential and keeping the large- $N$ limit in the scattering amplitudes. However, in the next and last section, we will not try to develop a perfect consistent computation but just to introduce a different pion effective mass in our previous calculation to check if a maximum is obtained for the bulk viscosity. Additionally, we have also checked that the use of the scattering amplitudes in the large- $N$ limit or the use of constant scattering amplitudes without any further correction give rise to similar results.

\section{THE CORNWALL-JACKIW-TOMBOULIS FORMALISM IN THE HARTREE APPROXIMATION}

In this section, we introduce a thermal pion mass from the effective potential obtained within the CJT formalism for the $\mathrm{L} \sigma \mathrm{M}$ in the Hartree approximation. Here we will refer to Ref. [44] where this approach is nicely presented. In a nutshell, the CJT method is a tool for the computation of an effective action, not only for the VEV of the field $\phi(x)=\langle\Phi(x)\rangle$, but also for the two-point function $G(x, y)=\langle T \Phi(x) \Phi(y)\rangle$. This generalized effective action can be understood as the generating functional of the twoparticle irreducible graphs (2PI). Some of the two-particle irreducible graphs which contribute to the CJT effective potential are shown in Fig. 19.

In Ref. [44], two different approaches were used to sum up certain sets of diagrams, namely the large- $N$ limit and the Hartree approximation. Of course, the large- $N$ approximation gives very close results to ours, and it gives no new information about the bulk viscosity. Therefore, we will consider the Hartree approximation in which only the "double bubble" diagram is taken into account, and which is equivalent to summing up all the "daisy" and "superdaisy" diagrams in the one-particle irreducible effective potential. The essential features of the method are sketched in Appendix C.
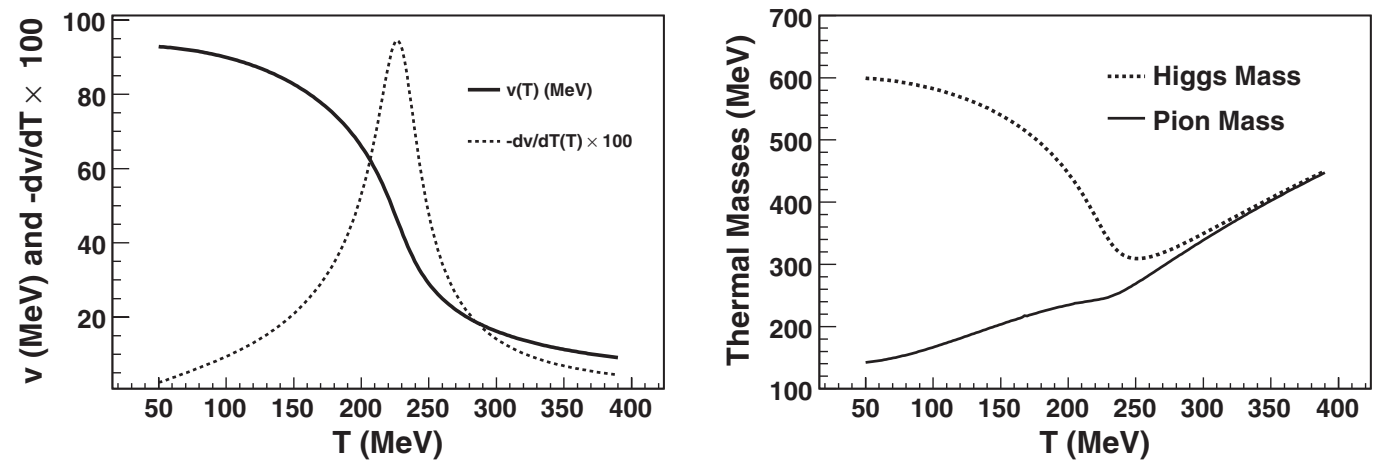

FIG. 13. Left panel: Order parameter and susceptibility in the CJT formalism in the Hartree approximation with masses $M_{\pi}=$ $138 \mathrm{MeV}$ and $M_{\sigma}=600 \mathrm{MeV}$ at $T=0$. Right panel: Thermal masses in the Hartree approximation for the same set of parameters. 

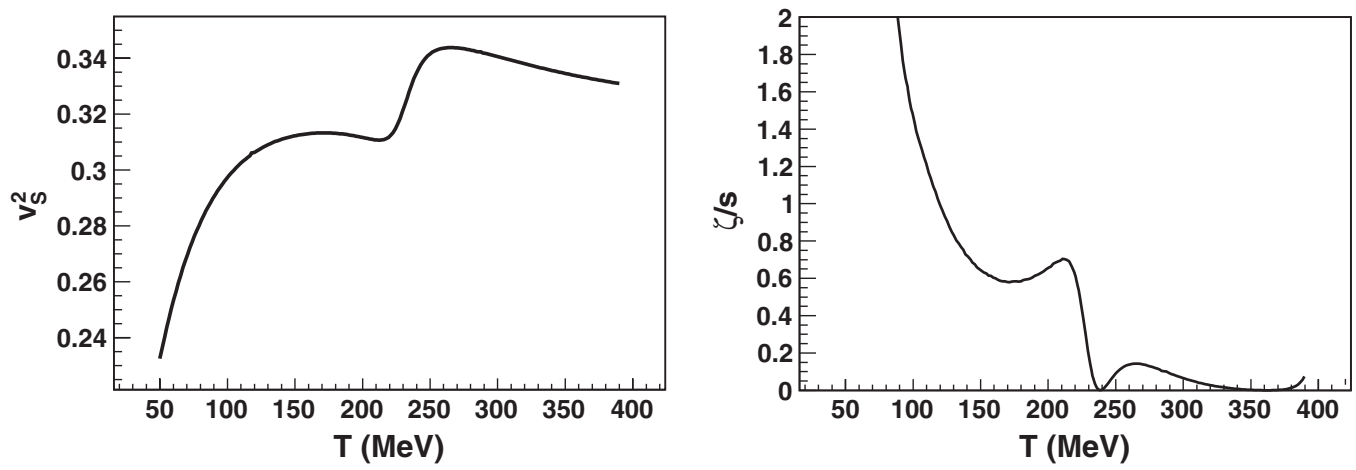

FIG. 14. Left panel: Square speed of sound for the case $M_{\pi}(T=0)=138 \mathrm{MeV}$ and $M_{\sigma}(T=0)=600 \mathrm{MeV}$. A local minimum is seen near the crossover temperature. Right panel: Bulk viscosity over entropy density. The first maximum appears at the local minimum of the speed of sound and the second maximum at the maximum of $v_{S}^{2}$, these points correspond to the zones where the loss of conformality is larger.

The effective potential is a function of the order parameter $\phi$ and two dressed propagators $G_{\sigma}$ and $G_{\pi}$, one for the Higgs and one for the pion, respectively. From the effective potential $V$, one can obtain the two gap equations,

$$
\frac{d V}{d G_{\pi}}=0 ; \quad \frac{d V}{d G_{\sigma}}=0
$$

and also minimize the effective potential with respect to the order parameter. If the dressed propagators are written as functions of some effective masses $G_{i}^{-1}=k^{2}+M_{i}^{2}$, then the three equations give the following nonlinear system for $\phi, M_{\sigma}$ and $M_{\pi}$ :

$M_{\sigma}^{2}=-2 \bar{\mu}^{2}+\frac{12 \lambda}{N} \phi^{2}+4 \lambda F\left(M_{\pi}\right)+\frac{12 \lambda}{N} F\left(M_{\sigma}\right)$,

$M_{\pi}^{2}=-2 \bar{\mu}^{2}+\frac{4 \lambda}{N} \phi^{2}+\frac{4(N+2) \lambda}{N} F\left(M_{\pi}\right)+\frac{4 \lambda}{N} F\left(M_{\sigma}\right)$,

$0=\left[-2 \bar{\mu}^{2}+\frac{4 \lambda}{N} \phi^{2}+\frac{12 \lambda}{N} F\left(M_{\sigma}\right)+4 \lambda F\left(M_{\pi}\right)\right] \phi-\epsilon$, where $F(M)$ is defined in Eq. (C24). This system can be solved numerically in order to obtain the effective masses and the order parameter which enter into our bulk viscosity calculation.

Of course, a renormalization program should be properly performed here. The technical aspects of the renormalization of the model can be found in Ref. [44] and in the references therein. However, in this work, we will deal only with the temperature-dependent part of the effective potential.

\section{A. Crossover case}

Within this new formalism, we start again by considering the crossover case with a Higgs mass of $600 \mathrm{MeV}$ and a pion mass of $138 \mathrm{MeV}$ at $T=0$. In Fig. 13, we present the results obtained by solving the nonlinear system in Eq. (71). In the left panel, we show the order parameter and its derivative (with opposite sign) with respect to the temperature. They look qualitatively very similar to the results found by using the large- $N$ limit with a crossover temperature of $T_{\mathrm{cr}}=230 \mathrm{MeV}$. In the right panel, we show the thermal masses, very similar also to those found

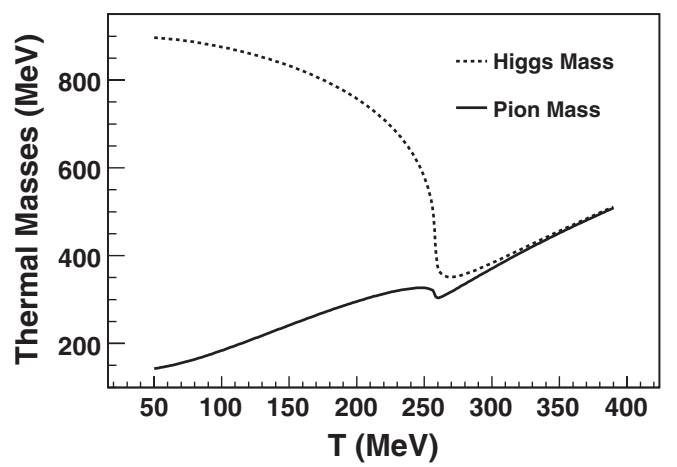

FIG. 15. Same as Fig. 13 but with $M_{\sigma}(T=0)=900 \mathrm{MeV}$. The order parameter goes faster toward zero, and the susceptibility clearly indicates the position of the crossover temperature. The pion mass has a zone of negative derivative near the crossover temperature. 

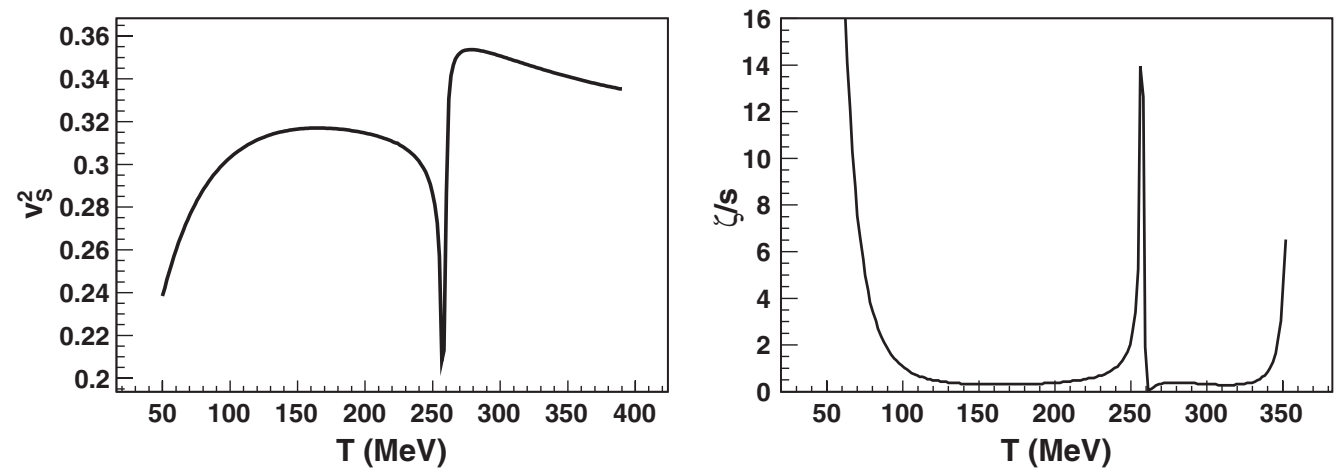

FIG. 16. Same as Fig. 14 but with $M_{\sigma}(T=0)=900 \mathrm{MeV}$. At $T_{\mathrm{cr}}$ the square speed of sound possesses an abrupt minimum showing a large violation of conformality. The bulk viscosity shows a clear maximum at $T_{\mathrm{cr}}$.

from the large- $N$ limit. However, one can see that the pion thermal mass shows a plateau at $T \simeq 220 \mathrm{MeV}$ with a derivative approaching zero in that region. This behavior produces an effect which is clearly seen in the speed of sound (where only the pion effects are included [45]) in the left panel of Fig. 14. Its shape is drastically different from the one coming from the large- $N$ limit computation. Now, a new minimum is obtained at $T=220 \mathrm{MeV}$, and it corresponds to a small loss of conformality around this region. The subsequent maximum is also a signature of the loss of conformality. Among them, the conformal value $1 / 3$ is reached. Therefore, one expects a zero bulk viscosity surrounded by two maxima. This is exactly the case in which the zero of the bulk viscosity resembles our zero in the large- $N$ limit, as we show in the right panel of the same figure.

Now, we can proceed to increase the Higgs mass. The solution of the nonlinear system in Eq. (71) is shown in Fig. 15, where a similar result to the previous case is obtained. The main difference is that the transition is more abrupt with a nice peak in the susceptibility. Now the curve shows a crossover temperature of $T_{\mathrm{cr}}=$ $260 \mathrm{MeV}$. The effect on the pion mass is seen in the right panel of that figure showing a clear zone around the $T_{\mathrm{cr}}$ where the pion mass possesses a negative derivative.

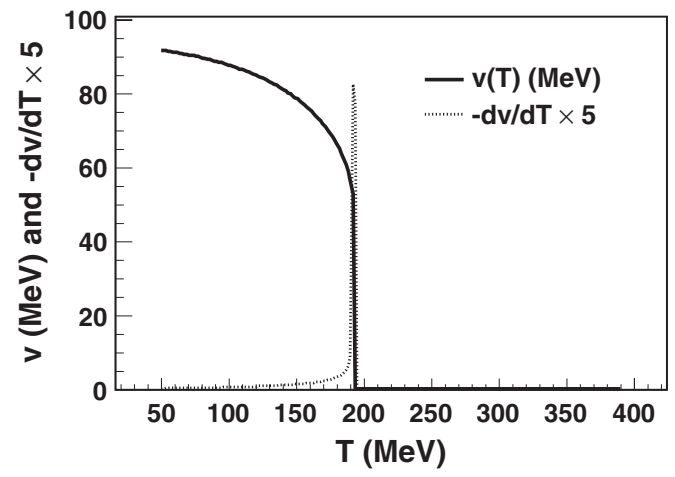

The implications of this behavior become clearer in the speed-of-sound curve which we plot in the left panel of Fig. 16. The previous minimum at $T_{\mathrm{cr}}$ is much deeper, showing a larger breaking of conformality. This minimum produces a peak in the bulk viscosity at the crossover temperature. This is the maximum of $\zeta / s$ which one expects at the phase transition and the one which is lost in the large- $N$ approximation.

\section{B. First-order phase transition in the chiral limit}

Finally, we take the chiral limit for pions at zero temperature. In the left panel of Fig. 17, we show how in the chiral limit the phase transition is of first order, with a clear discontinuity in the order parameter at $T_{c}=190 \mathrm{MeV}$. One feature of the Hartree approximation, as opposed to the large- $N$ limit, is that it does not respect the Goldstone theorem. This can be seen in the right panel of Fig. 17, where the pion mass in the low-temperature phase is different from zero. In addition, both masses show discontinuities at $T_{c}$. This jump is also seen in the speed of sound in the left panel of Fig. 18. The bulk viscosity presents a clear peak in the phase transition temperature with a finite discontinuity inherited by the first-order nature of the transition in the Hartree approximation.

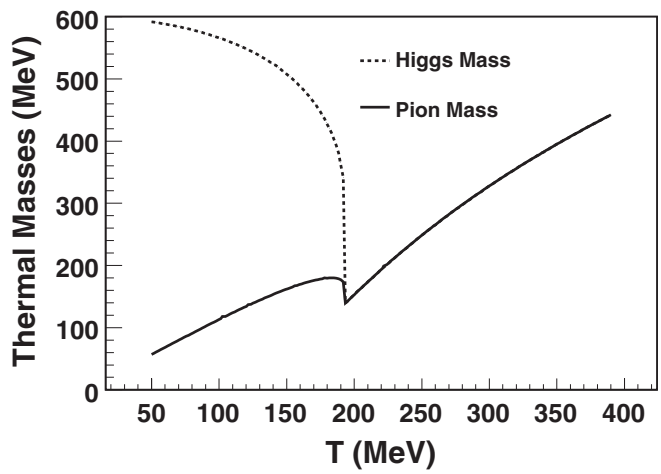

FIG. 17. Same as Fig. 13 but with $M_{\pi}(T=0)=0 \mathrm{MeV}$. The discontinuity of the order parameter reveals a first-order phase transition. In the right panel, one can observe that the Goldstone theorem is not satisfied for the pion mass within the Hartree approximation. 

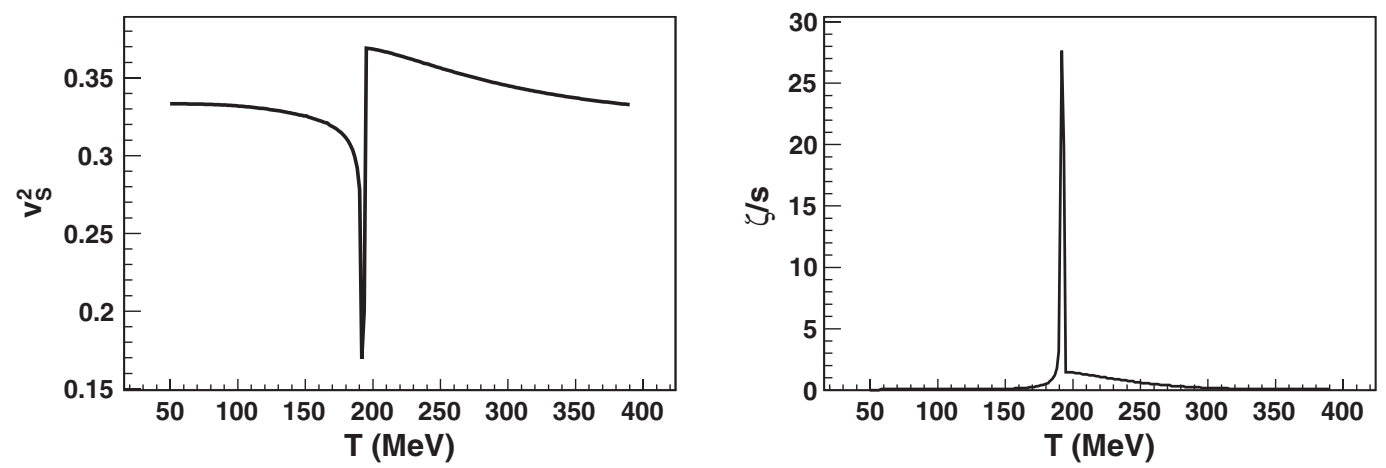

FIG. 18. Same as Fig. 14 but with $M_{\pi}(T=0)=0 \mathrm{MeV}$. The speed of sound inherits the discontinuity of the first-order transition. A maximum in the bulk viscosity over entropy density is still found at the critical temperature.

\section{SUMMARY}

In this work, we have extensively investigated the loss of conformality and the bulk viscosity in the $\mathrm{L} \sigma \mathrm{M}$. In the large- $N$ limit, where the dynamics of the Higgs field is washed out, the phase diagram has been calculated in order to pin down the location of the critical temperature. The bulk viscosity has been calculated in this limit, and we find that it vanishes at the conformal points, but it is different from zero otherwise. However, a maximum of the bulk viscosity is not found in the large- $N$ limit, consistent with other systems in the same limit as in Ref. [24]. Moreover, we find this result quite natural as recent dynamical renormalization group calculation shows that, in this limit, the bulk viscosity remains finite (it does not diverge) in the critical point [25].

However, we have shown that a maximum of the bulk viscosity can be found if one considers the Hartree approximation in the CJT effective potential. Just by obtaining the effective pion mass from this approach in our kinetic computation of the bulk viscosity, we find that a maximum is obtained at the crossover temperature for some appropriate region of the Higgs mass. Moreover, a clear maximum of $\zeta / s$ is obtained at the critical point when the pion mass is set to zero at $T=0$. However, this approximation fails to reproduce the Goldstone theorem of the $\mathrm{L} \sigma \mathrm{M}$ and gives rise to a first-order phase transition instead of a second-order one. Consequently, the large- $N$ and the Hartree approximations considered here seem to be relevant and informative to fully understand the behavior of the bulk viscosity of the $L \sigma \mathrm{M}$ at the critical point.

\section{ACKNOWLEDGMENTS}

We thank Felipe J. Llanes-Estrada, and Anna W. Bielska for reading the manuscript and for useful suggestions. We also thank the referee for helping us to improve the whole content of this manuscript. This work was supported by Grants No. Consolider-CSD2007-00042, No. FPA201127853-C02-01. and No. UCM-BSCH GR58/08 910309. Juan M. Torres-Rincon is a recipient of a FPU Grant from the Spanish Ministry of Education.

\section{APPENDIX A: $g_{0}\left(T, M^{2}\right), g_{1}\left(T, M^{2}\right)$ AND THEIR RELATION WITH THE MOMENTS OF $\boldsymbol{n}_{p}$}

The function $g_{0}\left(T, M^{2}\right)$ and its derivative $g_{1}\left(T, M^{2}\right)$ are needed for the temperature-dependent part of the effective potential in Eq. (33). The function $g_{0}\left(T, M^{2}\right)$ is defined as the following integral:

$$
g_{0}\left(T, M^{2}\right)=\frac{T^{4}}{3 \pi^{2}} \int_{y}^{\infty} d x\left(x^{2}-y^{2}\right)^{3 / 2} \frac{1}{e^{x}-1},
$$

where $y=M / T$. Note that in Eq. (33), $M^{2}$ corresponds to the function $G_{\pi}^{-1}[0, \chi]$. The notation $M^{2}$ is used to suggest that this factor will turn out to be the pion effective mass squared when the gap equation is solved.

The derivative of this function with respect to $M^{2}$, appearing in Eq. (34), defines the function $g_{1}(T, M)$ :

$$
g_{1} \equiv-\frac{d g_{0}}{d M^{2}}
$$

and can also be written as

$$
g_{1}\left(T, M^{2}\right)=\frac{T^{2}}{2 \pi^{2}} \int_{y}^{\infty} d x \frac{\sqrt{x^{2}-y^{2}}}{e^{x}-1} .
$$

In the limit $y \rightarrow 0$, i.e., the conformal limit as the pion mass goes to zero, the two functions are given by

$$
\begin{gathered}
g_{0}(T, 0)=\frac{T^{4}}{3 \pi^{2}} \Gamma(4) \zeta(4)=\frac{\pi^{2} T^{4}}{45}, \\
g_{1}(T, 0)=\frac{T^{2}}{2 \pi^{2}} \Gamma(2) \zeta(2)=\frac{T^{2}}{12} .
\end{gathered}
$$

These functions can be related to the moments of the Bose-Einstein distribution function, $n_{p}$ [46]. These moments read

$$
I^{\alpha_{1} \alpha_{2} \cdots \alpha_{n}}(T, m)=N \int \frac{d^{3} p}{(2 \pi)^{3} E_{p}} n_{p} p^{\alpha_{1}} p^{\alpha_{2}} \cdots p^{\alpha_{n}},
$$

with $E_{p}=\sqrt{p^{2}+m^{2}}$. They can be expanded in a tensor basis in terms of some coefficients $I_{n, k}$, depending on the temperature. These coefficients read 


$$
\begin{aligned}
I_{n, k}(T, m)= & \frac{N m^{n+2}}{(2 k+1) ! ! 2 \pi^{2}} \\
& \times \int_{1}^{\infty} d x x^{n-2 k}\left(x^{2}-1\right)^{k+1 / 2} \frac{1}{e^{y x}-1},
\end{aligned}
$$

where $x=E_{p} / m$ and $y=m / T$. They satisfy the recursion relation

$$
I_{n+2, k+1}(T, m)=\frac{1}{2 k+3}\left(I_{n+2, k}-m^{2} I_{n, k}\right),
$$

and can be related to some thermodynamical quantities in equilibrium, e.g., $P=I_{2,1}, \epsilon=I_{2,0}$, etc.

Performing a change of variables in Eqs. (A1) and (A3), one can express these two functions in terms of the $I_{n, k}$ integrals. In particular,

$$
\begin{aligned}
& g_{0}\left(T, M^{2}\right)=\frac{2}{N} I_{2,1}(T, M), \\
& g_{1}\left(T, M^{2}\right)=\frac{1}{N} I_{0,0}(T, M) .
\end{aligned}
$$

\section{APPENDIX B: $K^{i}, I^{i}$ AND THEIR RELATION WITH THE AUXILIARY MOMENTS OF $\boldsymbol{n}_{p}$}

In this work, we have used two different integration measures for the shear and bulk viscosities, respectively. These measures are naturally given by the form of the viscosities as an integration over the distribution function. In terms of the adimensional variables defined in Eq. (45), they explicitly read

$$
\begin{aligned}
& d \mu_{\eta}(x ; y)=d x\left(x^{2}-1\right)^{5 / 2} \frac{e^{y x}}{\left(e^{y x}-1\right)^{2}}, \\
& d \mu_{\zeta}(x ; y)=d x\left(x^{2}-1\right)^{1 / 2} \frac{e^{y x}}{\left(e^{y x}-1\right)^{2}},
\end{aligned}
$$

where, clearly, $x \in \Omega=[1, \infty)$. The moments of these measures are related to the respective source functions of the BUU equation. They have been defined as

$$
\begin{gathered}
K^{i}=\int_{\Omega} d \mu_{\eta} x^{i}, \\
I^{i}=\int_{\Omega} d \mu_{\zeta} x^{i} .
\end{gathered}
$$

From these measures, one can define the scalar products,

$$
\begin{aligned}
& \langle f \mid g\rangle_{\eta}=\int_{\Omega} d \mu_{\eta}(x ; y) f(x) g(x), \\
& \langle f \mid g\rangle_{\zeta}=\int_{\Omega} d \mu_{\zeta}(x ; y) f(x) g(x),
\end{aligned}
$$

and the corresponding norms in the usual way. In addition, one can define the corresponding orthogonal polynomial bases which expand the space generated by the $x^{i}$. For the shear viscosity, we use a monic orthogonal polynomial basis defined as

$$
\begin{gathered}
P_{0}(x)=1 \\
P_{1}(x)=x-\frac{K_{1}}{K_{0}} \\
P_{2}(x)=x^{2}+\frac{K_{0} K_{3}-K_{1} K_{2}}{K_{1}^{2}-K_{0} K_{2}} x+\frac{K_{2}^{2}-K_{1} K_{3}}{K_{1}^{2}-K_{0} K_{2}} \cdots .
\end{gathered}
$$

However, for the bulk viscosity, we follow the same convention except for the polynomial $P_{2}(x)$, which is fixed to be the source function in the left-hand side of Eq. (57),

$$
\begin{gathered}
P_{0}(x)=1, \\
P_{1}(x)=x, \\
P_{2}(x)=\left(\frac{1}{3}-v_{S}^{2}\right) x^{2}-\frac{1}{3}+\frac{T}{m} \frac{d m}{d T} v_{S}^{2} \cdots .
\end{gathered}
$$

Notice that now $\left\langle P_{1} \mid P_{2}\right\rangle=0$, but $\left\langle P_{0} \mid P_{2}\right\rangle$ is different from zero, so this basis is not completely orthogonal. Further information about the integration measures, scalar products, and polynomial basis can be found with more detail (also for the thermal conductivity coefficient, not studied here) in Ref. [47].

The two functions $K^{i}$ and $I^{i}$ are particular cases of more general coefficients expanding the "auxiliary moments" of the Bose-Einstein distribution function $n_{p}$ [46]. The auxiliary moments read

$\mathcal{J}^{\alpha_{1} \alpha_{2} \cdots \alpha_{n}}(T, m)=N \int \frac{d^{3} p}{(2 \pi)^{3} E_{p}} n_{p}\left(1+n_{p}\right) p^{\alpha_{1}} p^{\alpha_{2}} \cdots p^{\alpha_{n}}$

In an appropriate tensor basis, these moments can be expanded in terms of the coefficients $\mathcal{J}_{n, k}$ depending on the temperature. By using our adimensional variables, these coefficients can be written as

$$
\begin{aligned}
& \mathcal{J}_{n, k}(T, m) \\
& =\frac{N m^{n+2}}{(2 k+1) ! ! 2 \pi^{2}} \int_{1}^{\infty} d x x^{n-2 k}\left(x^{2}-1\right)^{k+1 / 2} \frac{e^{y x}}{\left(e^{y x}-1\right)^{2}} .
\end{aligned}
$$

Some of them can be related to thermodynamical quantities. For example,

$$
\frac{\mathcal{J}_{2,1}}{T}=n ; \quad \frac{\mathcal{J}_{3,1}}{T}=\epsilon+P ; \quad \frac{\mathcal{J}_{3,1}}{T^{2}}=s .
$$

The coefficients satisfy the recursion relation

$$
\mathcal{J}_{n+2, k+1}=\frac{1}{2 k+3}\left(\mathcal{J}_{n+2, k}-m^{2} \mathcal{J}_{n, k}\right) .
$$


It is not difficult to see that $K^{i}$ and $I^{i}$ are related with the $k=2$ and $k=0$ coefficients, respectively,

$$
\begin{gathered}
\mathcal{J}_{4+i, 2}=\frac{N m^{6+i}}{30 \pi^{2}} K_{i}, \\
\mathcal{J}_{i, 0}=\frac{N m^{2+i}}{2 \pi^{2}} I_{i} .
\end{gathered}
$$

Using the formula (B15), we can express the entropy density and the heat capacity in terms of the integrals $I^{i}$ :

$$
\begin{aligned}
s & =\frac{N m^{5}}{6 \pi^{2} T^{2}}\left(I_{3}-I_{1}\right), \\
C_{V} & =T \frac{\partial s}{\partial T}=\frac{N m^{5}}{2 \pi^{2} T^{2}}\left(I_{3}-\frac{d m}{d T} \frac{T}{m} I_{1}\right) .
\end{aligned}
$$

When there is no chemical potential, the temperature is the only independent variable, and the adiabatic speed of sound and the isochoric one coincide. Then, they can be written as

$$
v_{S}^{2}=\frac{s}{C_{V}}=\frac{1}{3} \frac{I_{3}-I_{1}}{I_{3}-\frac{d m}{d T} \frac{T}{m} I_{1}},
$$

which provides a universal relation for the speed of sound for any system which can be described in terms of free quasiparticles with masses depending only on the temperature and, in particular, for a free particle system.

\section{APPENDIX C: THE CORNWALL-JACKIW- TOMBOULIS EFFECTIVE POTENTIAL}

In this appendix, we briefly review the basic ingredients of the effective potential calculation in the context of the CJT formalism and the Hartree approximation. For more details, we refer the reader to Refs. [44,48-51]. To introduce this technique, we start with the derivation of the effective potential in the simple case of standard $\lambda \Phi^{4}$ theory at zero temperature, and, later, we will extend the calculation at finite temperature for the $\mathrm{L} \sigma \mathrm{M}$ in the Hartree approximation.

\section{1. $\lambda \Phi^{4}$ theory at zero temperature}

The classical Euclidean action for the $\lambda \Phi^{4}$ theory reads

$$
S[\Phi]=\int_{x}\left[\frac{1}{2} \partial^{\mu} \Phi(x) \partial_{\mu} \Phi(x)+\frac{m^{2}}{2} \Phi(x)^{2}+\frac{\lambda}{4 !} \Phi(x)^{4}\right],
$$

where we have defined

$$
\int_{x} \equiv \int d^{4} x
$$

In the context of the CJT method, the starting point is a generating functional of one- and two-point Green functions depending on a local source $J(x)$ and a bilocal one $K(x, y)$, defined as

$$
\begin{aligned}
Z[J, K]= & \int[d \Phi] \exp \left[S[\Phi]+\int_{x} J(x) \Phi(x)\right. \\
& \left.+\frac{1}{2} \int_{x, y} \Phi(x) K(x, y) \Phi(y)\right] .
\end{aligned}
$$

The corresponding generating functional for the connected Green functions, $\mathcal{W}[J, K]$, is defined as

$$
Z[J, K]=e^{W[J, K]} .
$$

The expectation value of the field $\phi(x)=\langle\Phi(x)\rangle_{J, K}$ and the connected two-point function $G(x, y)$ in the presence of the sources can be obtained through the functional derivatives of $\mathcal{W}[J, K]$.

$$
\begin{aligned}
& \frac{\delta \mathcal{W}[J, K]}{\delta J(x)}=\phi(x) ; \\
& \frac{\delta \mathcal{W}[J, K]}{\delta K(x, y)}=\frac{1}{2}[\phi(x) \phi(y)+G(x, y)] .
\end{aligned}
$$

This two-point function should not be confused with the tree-level propagator $D(x, y)$, whose inverse reads

$$
D^{-1}(x, y)=\frac{\delta^{2} S[\Phi]}{\delta \Phi(x) \delta \Phi(y)} .
$$

For the action given in Eq. (C1), this becomes

$$
D^{-1}(x, y)=-\left(\square_{x}-m^{2}-\frac{\lambda}{2} \Phi(x)^{2}\right) \delta(x-y) .
$$

The 2PI effective action can be obtained as the double Legendre transformation of $\mathcal{W}[J, K]$ :

$$
\begin{aligned}
\Gamma[\phi, G]= & \mathcal{W}[J, K]-\int_{x} \frac{\delta \mathcal{W}[J, K]}{\delta J(x)} J(x) \\
& -\int_{x, y} \frac{\delta \mathcal{W}[J, K]}{\delta K(x, y)} K(x, y),
\end{aligned}
$$

which, by using Eq. (C4), can be written as

$$
\begin{aligned}
\Gamma[\phi, G]= & \mathcal{W}[J, K]-\int_{x} \phi(x) J(x) \\
& -\frac{1}{2} \int_{x, y} K(x, y) \phi(x) \phi(y) \\
& -\frac{1}{2} \int_{x, y} G(x, y) K(y, x) .
\end{aligned}
$$

The stationary conditions for the 2PI effective action read

$$
\begin{aligned}
& \frac{\delta \Gamma[\phi, G]}{\delta \phi(x)}=-J(x)-\int_{y} K(x, y) \phi(y) ; \\
& \frac{\delta \Gamma[\phi, G]}{\delta G(x, y)}=-\frac{1}{2} K(x, y),
\end{aligned}
$$

which leads to the VEV and the dressed propagator when the sources are set to zero. 

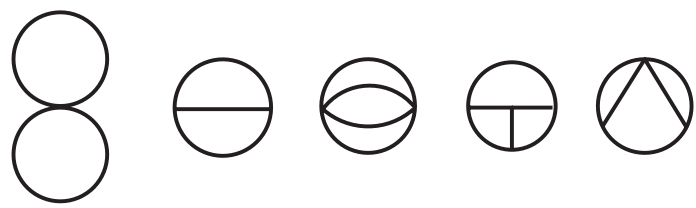

FIG. 19. Two-particle irreducible diagrams which enter in $\Gamma_{2}$. The solid lines correspond to dressed propagators $G$.

Following Cornwall-Jackiw-Tomboulis, the effective action can be written as [48]

$$
\begin{aligned}
\Gamma[\phi, G]= & S[\phi]-\frac{1}{2} \operatorname{Tr} \log G^{-1} \\
& -\frac{1}{2} \operatorname{Tr}\left(D^{-1} G-1\right)+\Gamma_{2}[\phi, G],
\end{aligned}
$$

where $\Gamma_{2}[\phi, G]$ is the sum of 2 PI diagrams with $G$ as internal propagators. Some of these diagrams can be seen in Fig. 19, and all of them contribute to the CJT effective action. In the following, we will refer to the first diagram as the double bubble diagram.

As usual, the effective potential (density) is obtained by assuming $\phi$ to be constant, so that one gets

$$
\begin{aligned}
V(\phi, G)= & U(\phi)+\frac{1}{2} \int_{k} \log G^{-1}(k) \\
& +\frac{1}{2} \int_{k}\left(D^{-1}(k) G(k)-1\right)+V_{2}[\phi, G],
\end{aligned}
$$

with $U(\phi)=m^{2} \phi^{2} / 2+\lambda \phi^{4} / 4$ ! being the classical potential.

Now, the equations of motion of the effective potential are obtained by minimizing $V$ with respect to $\phi$ and $G$ :

$$
\begin{aligned}
& \left.\frac{\delta V(\phi, G)}{\delta \phi}\right|_{\phi_{0}, G_{0}}=0, \\
& \left.\frac{\delta V(\phi, G)}{\delta G}\right|_{\phi_{0}, G_{0}}=0 .
\end{aligned}
$$

Here, the last equation provides the "mass gap equation" for $G_{0}$ in terms of $\phi$. Substituting this $G_{0}$ into Eq. (C11), one gets an effective potential in terms of $\phi$ only. Then, by minimizing this effective potential with respect to $\phi$, one obtains the order parameter (VEV) $\phi_{0}$.

In fact, Eq. (C13) is nothing but the Dyson-Schwinger equation for the propagator:

$$
G^{-1}=D^{-1}+\Sigma(\phi, G),
$$

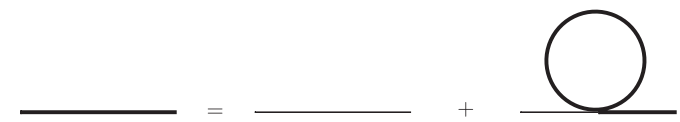

FIG. 20. Dyson-Schwinger equation for the dressed propagator $G$ (represented by wider solid line) in terms of the bare propagator $D$ (narrower solid line) and a self-energy insertion.

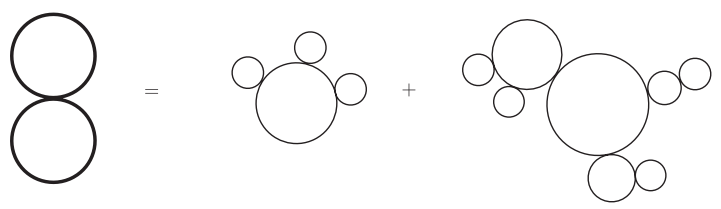

FIG. 21. In the Hartree approximation, only the 2PI diagram in the left-hand side is considered. It is equivalent to all daisy and superdaisy diagrams with the propagator $D$ as internal lines. We show some examples of those diagrams in the right-hand side.

where we have defined the self-energy as the functional derivative of the 2PI diagrams:

$$
\Sigma(\phi, G) \equiv 2 \frac{\delta V_{2}(\phi, G)}{\delta G} .
$$

On the other hand, it is possible to show that each 2PI diagram with $G$ as internal lines corresponds to an infinite number of one-particle irreducible diagrams with the bare propagator $D$ as internal lines.

As the most important example for our work here, we will consider the Hartree approximation for the calculation of $V_{2}$. Basically, it takes into account only the double bubble diagram of Fig. 19 [or, equivalently, diagrams $\mathcal{O}(\lambda)]$. Then, for the $\lambda \Phi^{4}$ theory, we have

$$
V_{2}=\frac{\lambda}{8}\left[\int_{k} G(k, \phi)\right]^{2},
$$

and the gap equation (C13) for the effective potential reads

$$
G^{-1}(k, \phi)=D^{-1}(k, \phi)+\frac{\lambda}{2} \int_{k} G(k, \phi),
$$

which is depicted in Fig. 20.

From this equation, one realizes that the double bubble diagram with $G$ as internal lines is equivalent to the full resummation of daisies and superdaisies diagrams in Fig. 21 with bare propagator as internal lines.

\section{2. $\mathrm{L} \sigma \mathrm{M}$ at finite temperature}

The extension of Eq. (C13) to the $\mathrm{L} \sigma \mathrm{M}$ with $N$ pions and a Higgs is straightforward. In the Hartree approximation, the graphs contributing to $V_{2}$ (and their numerical prefactors) are those of Fig. 22. Moreover, at finite temperature,

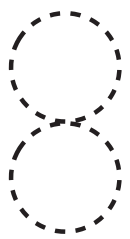

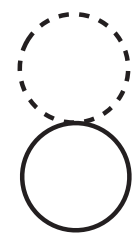

$2 N$

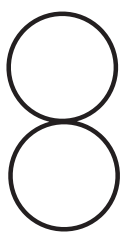

$N(N+2)$
FIG. 22. Double bubble diagrams contributing to the $V_{2}$ term of the effective potential in the Hartree approximation. Solid lines represent pions and dashed lines the $\sigma$. Their numerical prefactors in $V_{2}$ are also shown. 
the $k$ integration should be replaced by Matsubara summations. Defining

$$
\int_{\beta} f\left(i \omega_{n}, \mathbf{k}\right)=T \sum_{n} \int \frac{d^{3} k}{(2 \pi)^{3}} f\left(i \omega_{n}, \mathbf{k}\right),
$$

where $\omega_{n}=2 \pi T n$, the effective potential reads

$$
\begin{aligned}
V(\phi, G)= & U(\phi)+\frac{1}{2} \int_{\beta} \log G_{\sigma}^{-1}(k) \\
& +\frac{N}{2} \int_{\beta} \log G_{\pi}^{-1}(\phi, k) \\
& +\frac{1}{2} \int_{\beta}\left[D_{\sigma}^{-1}(k, \phi) G_{\sigma}(\phi, k)-1\right) \\
& +\frac{N}{2} \int_{\beta}\left[D_{\pi}^{-1}(k, \phi) G_{\pi}(\phi, k)-1\right) \\
& +V_{2}\left(\phi, G_{\pi}, G_{\sigma}\right),
\end{aligned}
$$

where $V_{2}$ is given by

$$
\begin{aligned}
V_{2}\left(\phi, G_{\sigma}, G_{\pi}\right)= & 3 \frac{\lambda}{N}\left[\int_{\beta} G_{\sigma}(\phi, k)\right]^{2} \\
& +N(N+2) \frac{\lambda}{N}\left[\int_{\beta} G_{\pi}(\phi, k)\right]^{2} \\
& +2 N \frac{\lambda}{N} \int_{\beta} G_{\sigma}(\phi, k) \int_{\beta} G_{\pi}(\phi, k) .
\end{aligned}
$$

The two gap equations are obtained by minimizing the effective potential with respect to $G_{\pi}$ and $G_{\sigma}$ :

$$
\begin{aligned}
G_{\sigma}^{-1}(\phi)= & D_{\sigma}^{-1}(\phi)+4 \lambda \int_{\beta} G_{\pi}(\phi)+\frac{12 \lambda}{N} \int_{\beta} G_{\sigma}(\phi), \\
G_{\pi}^{-1}(\phi)= & D_{\pi}^{-1}(\phi)+4(N+2) \lambda \int_{\beta} G_{\pi}(\phi) \\
& +\frac{4 \lambda}{N} \int_{\beta} G_{\sigma}(\phi) .
\end{aligned}
$$

To solve the system, one can use the ansatz for the dressed propagator: $G_{i}^{-1}=k^{2}+M_{i}^{2}$, with $M_{i}$ being the effective masses. Minimizing the effective potential (C19) with respect to $\phi$, one obtains the third equation which closes the nonlinear system leading to the three parameters $\phi$ (eventually called $v$ ), $M_{\sigma}$ and $M_{\pi}$. Introducing the explicit form of the bare and dressed propagators, the effective potential finally reads

$$
\begin{aligned}
V(\phi, M)= & -\bar{\mu}^{2} \phi^{2}+\frac{\lambda}{N} \phi^{4}-\epsilon \phi+Q\left(M_{\sigma}\right)+N Q\left(M_{\pi}\right) \\
& +\frac{1}{2}\left(-2 \bar{\mu}^{2}+\frac{12 \lambda}{N} \phi^{2}-M_{\sigma}^{2}\right) F\left(M_{\sigma}\right) \\
& -\frac{N}{2}\left(-2 \bar{\mu}^{2}+\frac{4 \lambda}{N} \phi^{2}-M_{\pi}^{2}\right) F\left(M_{\pi}\right) \\
& +3 \frac{\lambda}{N}\left[F\left(M_{\sigma}\right)\right]^{2}+(N+2) \lambda\left[F\left(M_{\pi}\right)\right]^{2} \\
& +2 \lambda F\left(M_{\sigma}\right) F\left(M_{\pi}\right),
\end{aligned}
$$

where

$$
\begin{aligned}
F(M) & =\int_{\beta} \frac{1}{k^{2}+M^{2}} \\
& =\int \frac{d^{3} k}{(2 \pi)^{3}} \frac{1}{2 E_{k}}+\int \frac{d^{3} k}{(2 \pi)^{3}} \frac{1}{E_{k}} \frac{1}{e^{\beta E_{k}}-1},
\end{aligned}
$$

and

$$
\begin{aligned}
Q(M) & =\frac{1}{2} \int_{\beta} \log \left(k^{2}+M^{2}\right) \\
& =\int \frac{d^{3} k}{(2 \pi)^{3}} \frac{E_{k}}{2}+T \int \frac{d^{3} k}{(2 \pi)^{3}} \log \left[1-e^{-\beta E_{k}}\right],
\end{aligned}
$$

with $E_{k}=\sqrt{k^{2}+M^{2}}$. As it is well known, both integrals have a $T=0$ (divergent) and a finite temperaturedependent part. The renormalization of the effective potential is discussed in Ref. [44] and references therein. In particular, there exists some difficulties in the renormalization under the Hartree approximation. See Refs. [52,53] for more details [54]. To our purposes, we only take into account the finite thermal contributions $F(M) \rightarrow F_{\beta}(M)$ and $Q(M) \rightarrow Q_{\beta}(M)$.
[1] A. Adare et al. (PHENIX Collaboration), Phys. Rev. Lett. 98, 162301 (2007).

[2] K. Aamodt et al. (ALICE Collaboration), Phys. Rev. Lett. 105, 252302 (2010).

[3] M. Luzum and P. Romatschke, Phys. Rev. C 78, 034915 (2008); 79, 039903(E) (2009).

[4] H. Song and U. W. Heinz, J. Phys. G 36, 064033 (2009).

[5] P. Bozek, J. Phys. G 38, 124043 (2011).
[6] P. K. Kovtun, D. T. Son, and A. O. Starinets, Phys. Rev. Lett. 94, 111601 (2005).

[7] L. P. Csernai, J. I. Kapusta, and L. D. McLerran, Phys. Rev. Lett. 97, 152303 (2006).

[8] A. Dobado, F. J. Llanes-Estrada, and J. M. Torres-Rincon, Phys. Rev. D 79, 014002 (2009).

[9] A. Dobado, F. J. Llanes-Estrada, and J. M. Torres-Rincon, Phys. Rev. D 80, 114015 (2009). 
[10] A. Dobado, F. J. Llanes-Estrada, and J. M. T. Rincon, AIP Conf. Proc. 1031, 221 (2008).

[11] S. Weinberg, Astrophys. J. 168, 175 (1971).

[12] V. Canuto and S. H. Hsieh, Nuovo Cimento Soc. Ital. Fis. 48B, 189 (1978).

[13] P. B. Arnold, C. Dogan, and G. D. Moore, Phys. Rev. D 74, 085021 (2006).

[14] D. Fernandez-Fraile and A. G. Nicola, Phys. Rev. Lett. 102, 121601 (2009).

[15] A. Dobado, F. J. Llanes-Estrada, and J. M. Torres-Rincon, Phys. Lett. B 702, 43 (2011).

[16] J. M. Torres-Rincon, Prog. Part. Nucl. Phys. 67, 461 (2012).

[17] A. Onuki Phys. Rev. E 55, 403 (1997).

[18] H. B. Meyer, Phys. Rev. Lett. 100, 162001 (2008).

[19] F. Karsch, D. Kharzeev, and K. Tuchin, Phys. Lett. B 663 , 217 (2008).

[20] K. Paech and S. Pratt, Phys. Rev. C 74, 014901 (2006).

[21] D. Kharzeev and K. Tuchin, J. High Energy Phys. 09 (2008) 093.

[22] B.-C. Li and M. Huang, Phys. Rev. D 80, 034023 (2009).

[23] P. Chakraborty and J. I. Kapusta, Phys. Rev. C 83, 014906 (2011).

[24] D. Fernandez-Fraile, Phys. Rev. D 83, 065001 (2011).

[25] E. Nakano, V. Skokov, and B. Friman, Phys. Rev. D 85, 096007 (2012).

[26] K. Dusling and T. Schafer, Phys. Rev. C 85, 044909 (2012).

[27] With respect to the Lagrangian in Ref. [9], we explicitly show the $N$ dependence of the coupling constant. The Lagrangian shown there was in Minkowski space.

[28] E. J. Weinberg and A.-q. Wu, Phys. Rev. D 36, 2474 (1987).

[29] J. Iliopoulos, C. Itzykson, and A. Martin, Rev. Mod. Phys. 47, 165 (1975).

[30] M. T. M. van Kessel, arXiv:0810.1412.

[31] J. Alexandre, V. Branchina, and J. Polonyi, Phys. Lett. B 445, 351 (1999).

[32] A. Dobado and J. Morales, Phys. Rev. D 52, 2878 (1995).

[33] G. Aarts and J. M. M. Resco, J. High Energy Phys. 02 (2004) 061.

[34] S. Jeon, Phys. Rev. D 52, 3591 (1995).

[35] E. M. Lifshitz and L.P. Pitaevskii, Physical Kinetics (Landau and Lifshitz Course of Theoretical Physics) (Pergamon, Oxford, 1981), Vol. 10.
[36] However, the component of $A(p)$ parallel to $x$ is not fixed by the BUU equation. If needed, it can be fixed by the Landau-Lifschitz condition

[37] The conformal value of the squared speed of sound depends on the space-time dimensions $D$ and reads

$$
\left.v_{S}^{2}\right|_{\mathrm{CFT}}=\frac{1}{D-1}
$$

[38] If the speed of sound is a monotonically increasing function with temperature, as the relativistic limit is $v_{S}^{2}=1$, this value can only be reached at $T \rightarrow \infty$.

[39] G. S. Denicol, T. Kodama, T. Koide, and P. Mota, Phys. Rev. C 80, 064901 (2009).

[40] A. Bazavov, T. Bhattacharya, M. Cheng, N. H. Christ, C. DeTar, S. Ejiri, S. Gottlieb, R. Gupta et al., Phys. Rev. D 80, 014504 (2009).

[41] S. Borsanyi, G. Endrodi, Z. Fodor, A. Jakovac, S. D. Katz, S. Krieg, C. Ratti, and K. K. Szabo, J. High Energy Phys. 11 (2010) 077.

[42] M. Laine and Y. Schroder, Phys. Rev. D 73, 085009 (2006).

[43] P. Gerber and H. Leutwyler, Nucl. Phys. B321, 387 (1989).

[44] N. Petropoulos, arXiv:0402136.

[45] In the Hartree approximation, the $\sigma$ is not $N$-suppressed anymore, and it should be included in the thermodynamical functions. However, given the similar results in Ref. [23], we will assume that the pertinent changes are minimal, and we do not include this degree of freedom in order to maintain the simplicity in the kinetic theory formalism.

[46] A. Muronga, Phys. Rev. C 76, 014910 (2007).

[47] J.M. Torres-Rincon, Ph.D. thesis, Universidad Complutense de Madrid, Spain, 2012, arXiv:1205.0782.

[48] J. M. Cornwall, R. Jackiw, and E. Tomboulis, Phys. Rev. D 10, 2428 (1974).

[49] G. Amelino-Camelia and S.-Y. Pi, Phys. Rev. D 47, 2356 (1993).

[50] G. Amelino-Camelia, Phys. Lett. B 407, 268 (1997).

[51] J. T. Lenaghan and D. H. Rischke, J. Phys. G 26, 431 (2000).

[52] J. Berges, S. Borsanyi, U. Reinosa, and J. Serreau, Ann. Phys. (Berlin) 320, 344 (2005).

[53] G. Fejos, A. Patkos, and Z. Szep, Nucl. Phys. A803, 115 (2008).

[54] We thank the referee for bringing these references to our attention. 